\title{
Observational Constraints on Local Lorentz Invariance ${ }^{1}$
}

\author{
Robert Bluhm \\ Department of Physics \\ Colby College \\ Waterville, ME 04901 USA
}

\begin{abstract}
The idea that local Lorentz invariance might be violated due to new physics that goes beyond the Standard Model of particle physics and Einstein's General Relativity has received a great deal of interest in recent years. At the same time, new experiments have been designed and conducted that are able to test Lorentz symmetry at unprecedented levels. Much of this theoretical and experimental progress has been driven by the development of the framework for investigating Lorentz violation known as the Standard Model Extension (SME). The SME is the lagrangian-based effective field theory that by definition contains all Lorentz-violating interaction terms that can be written as observer scalars involving particle fields in the Standard Model and gravitational fields in a generalized theory of gravity. This includes all terms that could arise from a process of spontaneous Lorentz violation as well as terms that explicitly break Lorentz symmetry. In this article, an overview of the SME is presented, including its motivations and construction. A very useful minimal version of the SME in Minkowski spacetime that maintains gauge invariance and power-counting renormalizability is constructed as well. Data tables summarizing tests of local Lorentz invariance for the different particle sectors in the Standard Model and with gravity are maintained by Kostelecký's group at Indiana University. A partial survey of these tests, including some of the high-precision sensitivities they attain, is presented here.
\end{abstract}

\footnotetext{
${ }^{1}$ Chapter to appear in The Springer Handbook of Spacetime, Springer-Verlag, 2013.
} 


\section{Contents}

1 Introduction $\quad 3$

2 Spacetime Symmetries in Relativity 4

2.1 Lorentz Transformations and Diffeomorphisms . . . . . . . . . . . 7

2.2 Particle and Observer Transformations . . . . . . . . . . . . . . . 9

2.3 Lorentz Violation . . . . . . . . . . . . . . . . . . . . . 11

3 Standard-Model Extension (SME) 13

3.1 Constructing the SME . . . . . . . . . . . . . . 16

3.2 Minimal SME . . . . . . . . . . . . . . . . . . 16

3.3 QED Extension . . . . . . . . . . . . . . . . 18

3.4 Extensions in Quantum Mechanics . . . . . . . . . . . . . . 19

3.5 Gravity Sector . . . . . . . . . . . . . . . . . 22

3.6 Spontaneous Lorentz Violation . . . . . . . . . . . . . . . . . . . . 24

4 Experimental Tests of Lorentz Violation 29

4.1 Data Tables . . . . . . . . . . . . . . . . . . . 32

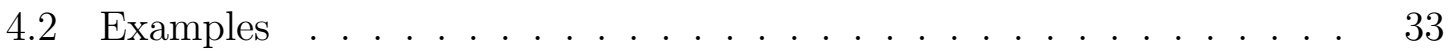

5 Summary and Conclusions $\quad 38$ 


\section{Introduction}

The Standard Model (SM) of particle physics and the theory of General Relativity (GR) are currently the best theories describing the four fundamental forces of nature: electromagnetism, the strong and weak nuclear forces, and gravity. There are no known experimental conflicts with predictions from either of these theories. Nonetheless, they are fundamentally different in that the SM is a quantum theory, while GR is a classical geometrical theory. It remains an open issue as to how to merge or reconcile the SM and GR into a unified theory that presumably contains a quantum description for gravity. The relevant scale for a quantum theory of gravity is typically taken as the Planck scale, which is approximately $10^{19} \mathrm{GeV}$. Promising candidates for a quantum theory of gravity include string theory and loop quantum gravity. These and other ideas for quantizing gravity can involve new features such as, for example, higher dimensions of space and time, brane-world scenarios, noncommutative geometries, and spacetime-varying fields or couplings. It is also possible that in merging GR into a quantum theory of gravity, the laws of relativity might not hold exactly at all energy scales.

Searching for experimental evidence of a quantum theory of gravity is challenging because conducting experiments at the Planck scale is not possible. However, suppressed effects emerging from a more fundamental theory might be observable in highly-sensitive low-energy experiments or in interferometry experiments with extremely long baselines. One candidate set of Planck-scale-suppressed signals is relativity violations associated with small breakings of local Lorentz symmetry. It has been shown, for example, that mechanisms arising in the context of string theory and other quantum theories of gravity might lead to violation of Lorentz symmetry. It is for this reason that considerable interest in the possibility of Lorentz violation has emerged in recent years, and a number of new high-precision experimental tests of local Lorentz invariance have been performed.

A key development in the investigation of Lorentz violation was the formulation of a comprehensive theoretical framework known as the Standard-Model Extension (SME) $[1,2,3,4,5]$. It contains both the SM and generalized theories of gravity (including GR) as well as all possible observer-independent Lorentz-violating interactions involving particle and gravitational fields. The SME has been used extensively to test for Lorentz violation in high-precision experiments. It also has theoretical features that are important for understanding the different types of processes that might lead to Lorentz violation. 
In this overview, the focus is on using the SME to investigate the possibility of Lorentz violation both theoretically and experimentally. An underlying assumption in using the SME is that at low energies (compared to the Planck scale) Lagrangian-based field theory gives what is currently the best description of elementary particles and their interactions. Therefore, if some new type of physics, such as Lorentz violation, goes beyond the SM and GR, then according to this assumption its leading-order corrections should be describable in the context of effective field theory. It is for this reason that the SME is suitable as a framework for investigating signals of Lorentz violation in experiments. However, in the search for a consistent quantum theory of gravity, it is also possible to consider ideas that fall outside the domain of Lagrangianbased field theory. These include ideas such as the breakdown of quantum mechanics, or where spacetime becomes discrete or noncontinuous at the quantum-gravity scale. Many of these ideas also lead to Lorentz violation. To the extent that they can be described at the level of effective field theory at low energies, then they should give rise to effects that are contained in the SME framework. However, if these alternative theories cannot be described using Lagrangian-based effective field theory, then it may not be possible to investigate their effects using the SME. In those cases, one would need to work within the context of the given model in order to investigate possible signals of Lorentz violation.

\section{Spacetime Symmetries in Relativity}

In special relativity, the equations of motion for particles and fields are invariant under Lorentz transformations. The Lorentz symmetry in this case is a global symmetry, with the transformations being the same at each point in the spacetime. The geometry of special relativity is that of a flat spacetime or Minkowski spacetime. In contrast, in GR, the effects of gravity are described by the curvature of spacetime, and the geometry is Riemannian. Lorentz symmetry still holds in GR, but only locally, e.g., in instantaneous infinitesimal inertial frames. In these local frames, called local Lorentz frames, the laws of special relativity are assumed to hold according to the equivalence principle. The symmetry in this case is local Lorentz invariance (LLI).

Curved spacetime in GR is described by the metric tensor, $g_{\mu \nu}$, the Riemann curvature tensor, $R_{\lambda \mu \nu}^{\kappa}$, and its contractions, including the Ricci tensor, $R_{\mu \nu}$, and the curvature scalar $R$. These quantities appear in the Einstein field equations along with the energy-momentum tensor for the matter fields, $T_{\mathrm{M}}^{\mu \nu}$, which acts as the source of 
the spacetime curvature. The Einstein equations are invariant under a set of spacetime transformations (defined mathematically in a later section) known as diffeomorphisms, which consist of mappings of the curved spacetime manifold back onto itself.

In particle physics described using special relativity, the matter fields often have additional symmetries, such as internal gauge symmetry or discrete spacetime symmetries. The latter include parity, $\mathrm{P}$, charge conjugation, $\mathrm{C}$, and time reversal, $\mathrm{T}$. In the SM of particle physics, many of these symmetries are broken either explicitly or through a process of spontaneous symmetry breaking. For example, spontaneous breaking of gauge symmetry is an essential feature of the Higgs mechanism in the electroweak model. In addition, all of the discrete symmetries C,P, and $\mathrm{T}$ are broken by the weak interactions, including the combination $\mathrm{CP}$, which is broken in certain meson interactions. However, a theorem in particle physics, known as the CPT theorem, states that the combination of all three of the discrete spacetime symmetries, CPT, must hold for all local interactions of point-like particles in the context of quantum field theory $[6,7,8,9]$. An essential assumption of the CPT theorem is that Lorentz symmetry must hold. This is important in investigations of Lorentz violation because it implies that if Lorentz symmetry is broken, then CPT breaking could occur as well because the conditions for the theorem to hold would not apply. This opens up another avenue of investigation of Lorentz violation in that high-precision tests of CPT symmetry can be used as well to test for Lorentz violation. Another theorem in the context of quantum field theory strengthens this connection. It states that in realistic effective field theories, interactions that break CPT also break Lorentz symmetry [10]. Evidently, there is a strong link between CPT violation and Lorentz violation, and any experiment looking for CPT violation can also be viewed as a Lorentz test in the context of quantum field theory.

The SM of particle physics can be be combined with GR to describe all four of the fundamental forces. This involves using a curved background with a metric $g_{\mu \nu}$ to describe the physical spacetime in which the SM particles move and interact. The resulting theory is a hybrid theory in which the SM fields are quantum fields with local $S U(3) \times S U(2) \times U(1)$ gauge symmetry. However, the metric field is not quantized, and the pure-gravity sector of the theory remains a classical theory. A classical Lagrangian can be written down for the full theory as a sum of a SM sector and a gravity sector,

$$
\mathcal{L}=\mathcal{L}_{\mathrm{SM}}+\mathcal{L}_{\mathrm{GR}}
$$

Derivatives of fields included in these expressions must be both gauge covariant and 
gravitationally covariant. The latter involves introduction of a spacetime connection $\Gamma_{\mu \nu}^{\lambda}$. The classical action of the theory is then given by the integral

$$
S=\int \sqrt{-g} \mathcal{L} d^{4} x
$$

where the factor involving the determinant of the metric, $g$, ensures that the spacetime volume element in the integral is covariant under general coordinate transformations. The Einstein field equations are obtained by variation of the action with respect to the metric. The SM fields appear in the Einstein equations by contributing to the energy-momentum tensor for the matter fields, $T_{\mathrm{M}}^{\mu \nu}$. Both the action and the field equations are invariant under diffeomorphism transformations.

In most particle-physics experiments, the gravitational interactions are irrelevant. In this case, the contributions from $\mathcal{L}_{\mathrm{GR}}$ are dropped and the metric is set equal to the Minkowski metric, $\eta_{\mu \nu}$. The theory can then be treated in the context of quantum field theory, where special relativity alone suffices. Without gravity, the relevant symmetries are global Lorentz symmetry and the local gauge symmetry of the SM, $S U(3) \times S U(2) \times U(1)$. However, with gravity included, the relevant symmetries of the theory change. Lorentz symmetry becomes a local symmetry, and diffeomorphism symmetry becomes important as well.

To observe the LLI of a theory in a curved spacetime, one approach is to make a coordinate transformation to a local Lorentz frame at each point in the spacetime manifold. In this way, the metric in the local coordinate system at each point reduces to $\eta_{\mu \nu}$, the connection vanishes, and locally the laws of special relativity apply. The choice of local Lorentz frame is not unique, however, since a Lorentz transformation at a given point leaves $\eta_{\mu \nu}$ unchanged.

An alternative approach keeps the spacetime frame fixed with metric $g_{\mu \nu}$, but also reveals the LLI at the same time. In this approach, four vector fields, $e_{\mu}{ }^{a}$, with labels $a=0,1,2,3$ are introduced. They are called vierbein or tetrad fields. They relate tensor components in the space-time frame (labeled by Greek indices) to the corresponding components in a local Lorentz frame (labeled by Latin indices). For example, the metric obeys

$$
g_{\mu \nu}=e_{\mu}{ }^{a} e_{\nu}{ }^{b} \eta_{a b}
$$

Since the metric is a symmetric field obeying $g_{\mu \nu}=g_{\nu \mu}$, it has at most ten independent degrees of freedom. In contrast, the vierbein, $e_{\mu}{ }^{a}$, has a total of sixteen independent degrees of freedom. The six extra degrees of freedom are associated with the LLI. 
There are several advantages to studying possible violations of LLI in a vierbein formalism. One is that fermions can more readily be introduced. In GR, particles form tensor representations under the group of linear transformations associated with general coordinate transformations, and there are no representations for spinhalf fermions. Thus, it is not possible to define Dirac gamma matrices or covariant derivatives of spinor fields in a spacetime manifold in GR. However, with a vierbein formalism it is possible to extend the usual definitions of these quantities in special relativity into curved spacetime. Another advantage of a vierbein formalism is that it allows the local Lorentz symmetry and diffeomorphism symmetry to be treated in a manner similar to local gauge symmetry in particle physics. However, to do this in a general way requires that an additional geometrical quantity called torsion be introduced into the theory. Geometrically, theories with torsion allow a twisting of coordinate axes as the axes are transported along a curve. This twisting cannot be described by the curvature tensor alone. The resulting geometry when torsion is included is called Riemann-Cartan geometry. (For reviews describing torsion and Riemann-Cartan geometry, see $[11,12])$. For these reasons, many investigations of Lorentz violation use a vierbein formalism and work in a generalized geometry, such as Riemann-Cartan geometry.

The use of a vierbein also involves introduction of a spin connection. It enters in covariant derivatives acting on local tensor components and plays the role of the gauge field for the Lorentz symmetry. In contrast, excitations of the metric field can be viewed as the gauge fields for the diffeomorphism symmetry. The relationship between the vierbein and spin connection is often a reflection of the type of spacetime geometry being considered. For example, in a Riemannian geometry (with no torsion), the spin connection is nondynamical and does not propagate. However, in a Riemann-Cartan geometry (with nonzero torsion), the spin connection must be treated as independent degrees of freedom that in principle can propagate. These different types of geometry can have effects on mechanisms that occur when Lorentz symmetry is violated. This is especially the case when Lorentz symmetry is spontaneously broken.

\subsection{Lorentz Transformations and Diffeomorphisms}

In special relativity, the Lorentz transformations consist of three rotations and three boosts. They are constant linear transformations that leave the Minkowski metric,

$\eta_{\mu \nu}$, invariant. Mathematically, they can be implemented by contracting the tensors in a theory with a transformation matrix $\Lambda_{\mu}{ }^{\alpha}$. In Cartesian coordinates, the 
transformation matrix obeys,

$$
\eta_{\mu \nu}=\Lambda_{\mu}^{\alpha} \Lambda_{\nu}^{\beta} \eta_{\alpha \beta}
$$

It is often useful to consider infinitesimal Lorentz transformations, which can be written as $\Lambda_{\mu}{ }^{\alpha} \simeq \delta_{\mu}{ }^{\alpha}+\epsilon_{\mu}{ }^{\alpha}$, where the six parameters, $\epsilon_{\mu}{ }^{\alpha}=-\epsilon_{\mu}^{\alpha}$, generate infinitesimal rotations and boosts. Under an infinitesimal particle Lorentz transformation, a tensor $T^{\lambda \mu \nu \cdots}$ transforms as,

$$
\begin{aligned}
T^{\lambda \mu \nu \cdots} & \rightarrow T^{\lambda \mu \nu \cdots}+\epsilon_{\rho}^{\lambda} T^{\rho \mu \nu \cdots} \\
& +\epsilon_{\rho}^{\mu} T^{\lambda \rho \nu \cdots}+\epsilon_{\rho}^{\nu} T^{\lambda \mu \rho \cdots}+\cdots
\end{aligned}
$$

In a theory with LLI, the action describing the theory and the equations of motion are left unchanged when all of the tensor fields in the theory are transformed by infinitesimal Lorentz transformations.

In the presence of gravity, the vierbein can be used to relate tensor components in a local Lorentz frame to the corresponding components in the space-time frame. A vierbein field appears for each tensor index. For example, for the tensor $T^{\lambda \mu \nu \cdots}$,

$$
T^{\lambda \mu \nu \cdots}=e_{a}^{\lambda} e_{b}^{\mu} e_{c}^{\nu} \cdots T^{a b c \cdots}+\cdots
$$

A local Lorentz transformation acts on the tensor components defined with respect to the local frame, e.g., $T^{a b c \cdots}$. For a local infinitesimal transformation, the six Lorentz parameters are written as $\epsilon_{a b}$. These depend on the spacetime coordinates at a given point. Under a local Lorentz transformation, the vierbein transforms as a vector,

$$
e_{\mu}^{a} \rightarrow e_{\mu}^{a}+\epsilon_{d}^{a} e_{\mu}^{d}
$$

Typically, in a gravitational theory with LLI, the six degrees of freedom associated with the local Lorentz symmetry are used to gauge away the six anti-symmetric components in the vierbein. The remaining ten components are symmetric and can be written in terms of field excitations $h_{\mu}{ }^{a}=h^{a}{ }_{\mu}$. For small excitations about a flat Minkowski background, the form of the vierbein can then be written as as

$$
e_{\mu}^{a}=\delta_{\mu}{ }^{a}+\frac{1}{2} h_{\mu}{ }^{a}
$$

Substituting this into Eq. 3 yields the usual expression for the metric in terms of small excitations about a Minkowski background, $g_{\mu \nu}=\eta_{\mu \nu}+h_{\mu \nu}$. 
Diffeomorphisms are mappings from one differentiable manifold to another. In GR, the mappings are from the spacetime manifold back to itself. Vectors and tensors transform in prescribed ways under diffeomorphisms, and diffeomorphism invariance in GR is the statement that the same physics is described by the spacetime manifold, metric, and matter fields both before and after a diffeomorphism is performed.

As with local Lorentz symmetry, diffeomorphism symmetry can be used to eliminate additional degrees of freedom. Under infinitesimal diffeomorphism transformations, points $x^{\mu}$ on the space-time manifold are mapped to neighboring points $x^{\mu}+\xi^{\mu}$, where the four parameters $\xi^{\mu}$ are spacetime dependent. Under infinitesimal diffeomorphisms, the metric transforms as

$$
g_{\mu \nu} \rightarrow g_{\mu \nu}-\partial_{\mu} \xi_{\nu}-\partial_{\nu} \xi_{\mu} .
$$

By gauge fixing the four diffeomorphism degrees of freedom, the metric can be reduced from ten down to six independent degrees of freedom. The excitations $h_{\mu \nu}$ then have six degrees of freedom as well after gauge fixing. These represent the six possible excitation modes for gravitational radiation that can occur in a generalized theory of gravity. For the case of Einstein's GR, the kinetic terms in the action are chosen so that four of these degrees of freedom do not propagate as physical modes and instead are called auxiliary modes. As a result, in Einstein's GR only two gravitational modes propagate, which are both massless transverse modes.

\subsection{Particle and Observer Transformations}

In investigations of possible Lorentz violation, it is important to distinguish between observer and particle transformations [2,3]. Observer transformations are essentially changes of coordinate systems, where the tensors describing particles and fields in the system are left physically unchanged. Lorentz transformations that transform between different local or global inertial frames are examples of observer transformations. Alternatively, Lorentz transformations can be performed directly on the tensor fields in a system, while leaving the observer frame (coordinate system) unchanged. When performed this way, the transformations are called particle transformations.

Similarly, in GR, general coordinate transformations can be performed, which correspond to a change of observer frame. These are observer transformations, which leave the equations of motion covariant in form. In contrast, diffeomorphisms are particle transformations performed with respect to a fixed observer, or in a fixed coordinate 
frame. The particles and fields of the system, including for example the metric, are transformed under diffeomorphisms in a prescribed way that leaves the equations of motion unchanged.

It is common to hear observer and particle transformations referred to, respectively, as passive and active transformations. In theories without spacetime symmetry breaking, these transformations are essentially inverses of each other in terms of how they act on tensor quantities. However, when a symmetry is broken, this is no longer the case, and it is important to make a clearer distinction between these two types of transformations.

It is reasonable to assert that a physical interaction should not depend on the choice of coordinates of a particular observer. As a result, any physical theory should be invariant under the relevant set of observer transformations for that theory. It is for this reason that observer transformations are not particularly meaningful as symmetry transformations. The physically important symmetry transformations are the particle transformations, which can be performed in a fixed arbitrary observer frame.

Even in a theory with interactions that break a spacetime symmetry, the resulting physical description should still not depend on any particular observer or choice of coordinates. Thus, in theories with broken Lorentz symmetry, the Lagrangian and equations of motion should be unchanged when an observer Lorentz transformation is performed. However, with Lorentz-violating interactions, the particle transformations are no longer symmetries of the theory. In a given observer frame, the physics can therefore change when a particle or field is transformed under a particle Lorentz transformation.

For example, consider a scattering experiment in special relativity. If Lorentz symmetry is violated, there may be preferred spatial orientations or speeds for the incoming and outgoing particles. As a result, particles scattered in different directions or with different speeds, with respect to a given observer, may behave differently. Nonetheless, the theory remains fully observer-independent. If a different observer measures the same scattering events, the resulting physical effects will be unaffected. All that happens in an observer Lorentz transformation is that the same physical events are expressed with respect to a different Lorentz frame.

According to this approach, when LLI is broken, it is only the particle Lorentz transformations that are broken. The theory remains Lorentz observer-independent at all times. Likewise, in an extension of GR that incorporates spacetime symmetry 
breaking, the relevant transformations are particle local Lorentz transformations and diffeomorphisms. If either of these are broken, the theory should still be covariant under observer local Lorentz transformations and observer general coordinate transformations.

\subsection{Lorentz Violation}

Lorentz symmetry is fundamental in both the SM and in GR. It should therefore be tested as accurately as possible as a way of testing the validity of these theories. In addition, it has been shown in the context of quantum-gravity theories that small violations of Lorentz symmetry might occur. For example, in string field theory mechanisms can occur that might lead to spontaneous breaking of Lorentz symmetry $[13,14,15,16,17,18,19]$. Indeed, it was this idea that led to the development of the SME, which in turn has stimulated a variety of new experimental tests of LLI. (For reviews of various experimental and theoretical approaches to Lorentz and CPT violation see $[20,21,22,23])$.

In string field theory, a string state can be expanded as a sum of tensor-valued particle states, where the particle masses increase with the order of the tensor. String interactions provide couplings between the particle states. Spontaneous Lorentz violation can occur in this context when a string field theory has a nonperturbative vacuum that can lead to one or more of the tensor-valued fields, $T$, acquiring nonzero vacuum expectation values or vevs, $\langle T\rangle \neq 0$. When this occurs, the low-energy effective theory can contain terms of the form

$$
\mathcal{L} \sim \frac{\lambda}{m_{P}^{k}}\langle T\rangle \Gamma \bar{\psi}(i \partial)^{k} \chi,
$$

where $k$ is an integer power, $\lambda$ is a coupling constant, $\Gamma$ is a generalized Dirac matrix, $m_{P}$ is the Planck mass, and $\psi$ and $\chi$ are fermion fields. Note that the higherdimensional $(k>0)$ derivative couplings are expected to be balanced by additional inverse factors of the Planck mass $m_{P}$.

In this expression, the tensor vev, $\langle T\rangle$, carries spacetime indices, which are not written out in this notation. This vev is effectively a set of background functions or constants that are fixed in a given observer frame. As tensor-valued backgrounds, these coefficients can have preferred directions in spacetime or velocity dependence. In other words, they induce Lorentz violation. A more general interaction term can 
be defined by absorbing all of the couplings and inverse mass factors into the vev. The Lorentz-violating interactions then have the form

$$
\mathcal{L} \sim t^{(k)} \Gamma \bar{\psi}(i \partial)^{k} \chi
$$

where the coefficients $t^{(k)}$ carry spacetime indices and act as fixed Lorentz-violating background fields. In addition to interactions with fermions, additional terms involving gauge-field couplings and gravitational interactions are possible as well.

The SME is a generalization of these types of interactions to include all possible contractions of known SM and gravitational fields with fixed background coefficients $t^{(k)}[1,2,3,4,5]$. This includes all arbitrary-dimension interaction terms inducing Lorentz violation in effective field theory. The coefficients for Lorentz violation, $t^{(k)}$, are examples of SME coefficients. They are assumed to be heavily suppressed, presumably by inverse powers of the Planck mass. In fact, since no Lorentz violation has been observed in nature, it must be that these SME coefficients are small.

By developing the SME in this generalized way, a framework that is particularly well suited for phenomenology results. In this approach, the Lorentz-violating SME coefficients are treated as quantities to be bounded in experiments. They can be thought of as vevs arising in a process of spontaneous Lorentz violation or simply as being due to explicit Lorentz violation from some unknown mechanism.

The interactions in 10 can also be used to study other processes related to Lorentz and CPT violation. For example, terms of this form have been shown to induce a form of CPT-violating baryogenesis [24].

Another example of Lorentz violation comes from noncommutative field theory [25]. These are theories with noncommuting coordinates $\left[x^{\mu}, x^{\nu}\right]=i \theta^{\mu \nu}$. It has been shown that this type of geometry can occur naturally in string theory and that it leads to Lorentz violation $[26,27,28,29,30]$. The fixed parameters $\theta^{\mu \nu}$ break Lorentz symmetry and act effectively as fixed background tensors. For example, in an effective field theory with a $U(1)$ gauge field in a noncommutative geometry, interaction terms of the form

$$
\mathcal{L} \sim i q \theta^{\alpha \beta} F_{\alpha \beta} \bar{\psi} \gamma^{\mu} D_{\mu} \psi
$$

can arise. Here, $F_{\alpha \beta}$ is the field strength, $q$ is the charge, and $D_{\mu}$ is a gauge-covariant derivative. As in Eq. (12) the interaction takes the form of a scalar-valued product of known particle fields, derivative operators, and a set of fixed background functions. It is straightforward to write these interactions in terms of SME couplings. 
There are a number of other examples of theories with Lorentz violation that have been put forward in recent years. These include models with spacetime-varying fields, quantum gravity models, multiverses, and braneworld scenarios. See, for example, $[31,32,33,34,35,36,37,38,39,40,41,42,43]$. It is also possible to construct models with specific types of Lorentz violation. These include models that maintain spatial rotational invariance while breaking only boost transformations, models with Lorentz-violating dispersion relations constructed using higher-order derivative interactions, and vector-tensor models in gravity that spontaneously break Lorentz symmetry. To the extent that these types of models can be described wholly or in part using Lagrangian-based effective field they, they can be investigated using the SME. However, some ideas for quantum-gravity theories contain new features that are not readily described in the context of effective field theory. Examples include ideas such as spacetime foam, causal sets, and relative locality. See, for example, [44, 45, 46, 47]. In these types of models, many of the signals of Lorentz violation that arise are not suitable for investigation using the SME and instead must be studied in the context of the specific theory.

A number of phenomenological frameworks involving certain kinds of Lorentz violation have been used by experimentalists in the past. These include the RobertsonMansouri-Sexl framework and the PPN formalism [48, 49, 50]. In some cases, these and other theories describe parameterized equations of motion or dispersion relations that do not originate from a scalar Lagrangian. However, to the extent that these models can be described by effective field theory defined by a scalar Lagrangian, they are compatible with the SME and direct links between their parameterizations and the SME coefficients can be obtained. Since CPT violation in field theory is associated with Lorentz violation, it follows as well that any observer-independent effective field theory describing CPT violation should also be contained within the SME. Since CPT can be tested to very high precision in experiments comparing matter and antimatter, this type of experiment is also ripe as a testing ground for Lorentz violation.

\section{$3 \quad$ Standard-Model Extension (SME)}

Currently, there is no consistent quantum theory of gravity that can be used in detailed examinations of the phenomenology of Lorentz violation at accessible energies. Nonetheless, progress can still be made using effective field theory. To be realistic, an effective field theory must contain the SM and a theory of gravity (such as GR), and it 
must be compatible with observations. It must also maintain observer independence. The Standard-Model Extension (SME) is defined to be the most general effective field theory of this type incorporating arbitrary observer-independent Lorentz violation.

The SME lagrangian by definition contains all observer-scalar terms that consist of products of SM and gravitational fields with each other as well as with additional couplings that introduce violations of Lorentz symmetry. In principle, there are an infinity of terms in the SME, including nonrenormalizable terms of arbitrary dimension. Most of these terms are expected to be suppressed by large inverse powers of the Planck scale. The question of how to extract a useful finite subset of terms from the full SME to analyze a given experiment becomes relevant, and there are a number of different ways to proceed. Perhaps the most natural approach is to follow the direction indicated by the experiments testing Lorentz violation. While these tend to be highly interdisciplinary, and include experiments in astrophysics, gravity, atomic, nuclear, and particle physics, as well as laboratory experiments with macroscopic media and space-based tests, several primary divisions and classifications can be made.

One important split is between experiments that can ignore the effects of gravity from those that cannot. For this reason, a distinction is made between limits of the SME that do not include gravity (where special relativity and global Lorentz invariance are paramount) from those where gravity is included (where Lorentz symmetry acts as a local symmetry in a curved spacetime). It is expected that the nongravitational limits of the SME will in general be subsets of larger SME limits that include gravity. For example, if the curvature is set to zero and the metric is replaced by the Minkowski metric, an SME limit with gravity should reduce to a corresponding SME limit in which gravity is excluded. Starting from the ground up in constructing explicit limits of the SME, it is therefore natural to ignore gravity at first and then to generalize the resulting theories to incorporate gravity.

In the absence of gravity, a second primary division between subsets of the SME can be made based on the types of SM fields and interactions (especially their dimensionality) that are included. Since the SM itself is a renormalizable and gauge-invariant theory, a first step in constructing a useful SME limit is to incorporate Lorentz violation while maintaining these features. This limit restricting the SME to power-counting renormalizable and gauge-invariant terms is called the minimal SME (mSME). An advantage of working with the mSME is that each particle sector has a finite independent set of mSME coefficients that can be probed experimentally. Indeed, in recent years, experimentalists have adopted using bounds on mSME coefficients as 
the primary means of reporting sensitivity of their experiments to Lorentz violation. Many of the low-energy experiments testing Lorentz violation involve only electromagnetic interactions between charged particles and photons. For this reason, it is useful as well to define a minimal QED sector of the SME. In a field theory with charged fermions, the minimal QED lagrangian consists of the standard Dirac and Maxwell terms supplemented by Lorentz-violating terms that maintain U(1) gauge symmetry and power-counting renormalizability.

If leading order effects are of primary interest, then SME limits at the level of relativistic quantum mechanics can be constructed. This is particularly useful in investigations of low-energy atomic systems, where small corrections to atomic energy levels can result from Lorentz breaking at leading order. Experiments using particle traps, masers, and high-precision spectroscopy can then be analyzed in a straightforward manner using perturbation theory.

On the other hand, if first-order effects can be ruled out in an experiment, it will be necessary to construct limits of the SME that include nonrenormalizable terms. In some scenarios for Lorentz violation, it might happen that Lorentz violation only stems from terms of dimension greater than four in the lagrangian. Alternatively, if the SME coefficients at leading order are known to have experimental bounds at levels suppressed by two powers of the Planck scale, then it becomes appropriate to look for signals of Lorentz violation at subleading order as well. For these reasons, limits of the SME that contain higher-dimension nonrenormalizable terms are of interest.

In some experiments, particular types of particle behaviors play a major role in attaining sensitivity to Lorentz violation. Examples include spin-precession effects, interference, or flavor-changing oscillations. In these situations it can be advantageous to build specific types of models out of subsets of the SME. Such models can then be used as frameworks for phenomenology. It is also useful to consider complementary tests of Lorentz violation when different experiments only have sensitivity to combinations of SME experiments. An example of this involves CPT tests. These experiments with particles and antiparticles are typically sensitive at leading order to combinations of the CPT-odd terms in the SME. At the same time, different experiments with the particles alone might have leading-order sensitivity to different combinations of both CPT-even and CPT-odd terms. However, by analyzing both sets of experiments in terms of SME coefficients in a complementary manner, it becomes possible to place more stringent bounds on individual types of Lorentz violation. 


\subsection{Constructing the SME}

The SME contains the SM, a gravity sector, and all possible observer-independent interactions of these conventional fields with fixed Lorentz-violating backgrounds, which are referred to as SME coefficients. As is typically done in field theory, the SME can be constructed in terms of a Lagrangian. The equations of motion are then obtained by variations of the action with respect to the fundamental fields in the theory. The SME Lagrangian has three primary sectors, including one for the SM, one for gravity, and a Lorentz-violating sector,

$$
\mathcal{L}_{\mathrm{SME}}=\mathcal{L}_{\mathrm{SM}}+\mathcal{L}_{\mathrm{GRAV}}+\mathcal{L}_{\mathrm{LV}}
$$

The full SME with gravity is defined using a vierbein formalism. This permits a natural distinction between the spacetime manifold and local Lorentz frames.

The observer independence of the SME requires that all of the terms in the Lagrangian be observer scalars under both general coordinate transformations and local Lorentz transformations. This means that every spacetime index and every local Lorentz index must be fully contracted in the lagrangian.

The SME is not invariant under particle diffeomorphisms and particle local Lorentz transformations. The four infinitesimal parameters $\xi^{\mu}$ comprise the diffeomorphism degrees of freedom, while the six infinitesimal parameters $\epsilon_{a b}=-\epsilon_{b a}$ carry the six Lorentz degrees of freedom. In total, there are ten relevant spacetime symmetries. Violation of these symmetries occurs when an interaction term in the Lagrangian contains SME coefficients that remain fixed under a particle local Lorentz transformation or diffeomorphism.

\section{$3.2 \quad$ Minimal SME}

Since the SM works remarkably well to describe nongravitational particle interactions at accessible energies, it makes sense initially to construct a minimal extension beyond the SM that contains only those interactions for which experiments are likely to have the greatest sensitivity. These are the interactions that break LLI while maintaining all of the other desirable features of the SM, such as gauge invariance and renormalizability. The mSME is the restriction of the SME to these power-counting renormalizable and gauge-invariant terms in the absence of gravity. 
The mSME Lagrangian can be separated into Lorentz-invariant and Lorentz- and CPT-violating parts:

$$
\mathcal{L}_{\mathrm{SME}, \min }=\mathcal{L}_{\mathrm{SM}}+\mathcal{L}_{\mathrm{LV}, \min }
$$

The Lorentz-invariant sector is identified with the usual Lagrangian for the minimal SM. The Lorentz-violating Lagrangian is the restriction to terms of mass dimension 3 and 4 in $\mathcal{L}_{\mathrm{LV}}$ that maintain $\mathrm{SU}(3) \times \mathrm{SU}(2) \times \mathrm{U}(1)$ gauge symmetry.

The first component, $\mathcal{L}_{\mathrm{SM}}$, describes the usual interactions for the strong and electroweak interactions. The matter fields consist of three generations of quarks and leptons. These interact through exchange of gauge fields. A Higgs sector is needed to provide mass terms for the $\mathrm{W}$ and $\mathrm{Z}$ bosons in the weak interactions through the Higgs mechanism, and Yukawa couplings are needed to give the quarks and leptons mass terms as well. The Lagrangian $\mathcal{L}_{\mathrm{SM}}$ can be split into five parts corresponding to these different sectors:

$$
\begin{aligned}
\mathcal{L}_{\mathrm{SM}}= & \mathcal{L}_{\text {lepton }}+\mathcal{L}_{\text {quark }}+\mathcal{L}_{\text {Yukawa }} \\
& +\mathcal{L}_{\text {Higgs }}+\mathcal{L}_{\text {gauge }}
\end{aligned}
$$

For illustration, the form of the terms for the lepton sector are given here:

$$
\mathcal{L}_{\text {lepton }}=\frac{1}{2} i \bar{L}_{A} \gamma^{\mu} \stackrel{\leftrightarrow}{D_{\mu}} L_{A}+\frac{1}{2} i \bar{R}_{A} \gamma^{\mu} \stackrel{\leftrightarrow}{D_{\mu}} R_{A}
$$

In this notation, the left- and right-handed lepton multiplets are denoted as

$$
L_{A}=\left(\begin{array}{c}
\nu_{A} \\
l_{A}
\end{array}\right)_{L} \quad, \quad R_{A}=\left(l_{A}\right)_{R}
$$

The index $A=1,2,3$ labels the three flavors, with $l_{A}=(e, \mu, \tau)$ denoting the electron, muon, and tau particles, and $\nu_{A}=\left(\nu_{e}, \nu_{\mu}, \nu_{\tau}\right)$ labeling the three corresponding neutrinos. The gauge-covariant derivative is denoted $D_{\mu}$, and the notation $A \stackrel{\leftrightarrow}{\partial_{\mu}} B \equiv A \partial_{\mu} B-\left(\partial_{\mu} A\right) B$ is adopted. For the remaining terms in $\mathcal{L}_{\mathrm{SM}}$, see $[2,3]$.

The Lorentz- and CPT-violating part of the Lagrangian $\mathcal{L}_{\mathrm{LV} \text {,min }}$ can also be written as a sum of terms distinguishing the contributions from the lepton, quark, Yukawa, Higgs, and gauge sectors. These partial lagrangians can be further separated into CPT-even and CPT-odd parts. Each of these terms consist of contractions of the SM fields with SME coefficients. 
To illustrate for the lepton sector, the Lorentz-violating terms are:

$$
\begin{aligned}
\mathcal{L}_{\text {lepton }}^{\text {CPT-even }}= & \frac{1}{2} i\left(c_{L}\right)_{\mu \nu A B} \bar{L}_{A} \gamma^{\mu} \stackrel{\leftrightarrow}{D^{\nu}} L_{B} \\
+ & +\frac{1}{2} i\left(c_{R}\right)_{\mu \nu A B} \bar{R}_{A} \gamma^{\mu} \stackrel{\leftrightarrow}{D^{\nu}} R_{B} \\
\mathcal{L}_{\text {lepton }}^{\text {CPT-odd }}= & -\left(a_{L}\right)_{\mu A B} \bar{L}_{A} \gamma^{\mu} L_{B} \\
& -\left(a_{R}\right)_{\mu A B} \bar{R}_{A} \gamma^{\mu} R_{B} .
\end{aligned}
$$

In these expressions, the SME coefficients $a_{\mu}$ have dimensions of mass, while $c_{\mu \nu}$ are dimensionless and traceless. It is these quantities that act as fixed background fields under particle Lorentz transformations and induce the breaking of Lorentz symmetry.

\subsection{QED Extension}

The QED limit of the SME is useful for specific applications involving charged particle and photon interactions. It contains the leading-order Lorentz- and CPT-violating terms that maintain $\mathrm{U}(1)$ gauge invariance. For a single Dirac fermion $\psi$ of mass $m$ the lagrangian is $\mathcal{L}_{\mathrm{QED} \text {,min }}=\mathcal{L}_{\text {fermion }}+\mathcal{L}_{\text {photon }}$. The fermion-sector piece can be written as

$$
\mathcal{L}_{\text {fermion }}=\frac{1}{2} i \bar{\psi} \Gamma^{\mu} \stackrel{\leftrightarrow}{D_{\mu}} \psi-\bar{\psi} M \psi
$$

where the gauge-covariant derivative is $D_{\mu}=\partial_{\mu}+i q A_{\mu}$ and $\Gamma^{\nu}$ and $M$ are defined by

$$
\begin{gathered}
\Gamma^{\nu}=\gamma^{\nu}+c^{\mu \nu} \gamma_{\mu}+d^{\mu \nu} \gamma_{5} \gamma_{\mu}+e^{\nu} \\
+i f^{\nu} \gamma_{5}+\frac{1}{2} g^{\lambda \mu \nu} \sigma_{\lambda \mu}, \\
M=m+a_{\mu} \gamma^{\mu}+b_{\mu} \gamma_{5} \gamma^{\mu}+\frac{1}{2} H^{\mu \nu} \sigma_{\mu \nu} .
\end{gathered}
$$

These equations contain the usual QED terms for a single fermion. The nonstandard terms violate Lorentz symmetry, and most have analogues in the minimal SME. However, the dimensionless coefficients $e^{\nu}, f^{\nu}, g^{\lambda \mu \nu}$ have no analogue in the minimal SME because they are incompatible with $\mathrm{SU}(2) \times \mathrm{U}(1)$ symmetry. They are included in the minimal QED extension because they are compatible with $\mathrm{U}(1)$ invariance and could emerge from terms in the effective action involving the Higgs field. 
The lagrangian in the photon sector is

$$
\begin{aligned}
\mathcal{L}_{\text {photon }}= & -\frac{1}{4} F_{\mu \nu} F^{\mu \nu}-\frac{1}{4}\left(k_{F}\right)_{\kappa \lambda \mu \nu} F^{\kappa \lambda} F^{\mu \nu} \\
& +\frac{1}{2}\left(k_{A F}\right)^{\kappa} \epsilon_{\kappa \lambda \mu \nu} A^{\lambda} F^{\mu \nu}
\end{aligned}
$$

For simplicity here, any total-derivative terms are neglected, as is a possible term of the form $\left(k_{A}\right)_{\kappa} A^{\kappa}$. Some discussion of the latter can be found in Refs. [2,3].

In these expressions, the terms with coefficients $a_{\mu}, b_{\mu}, e_{\mu}, f_{\mu}, g_{\lambda \mu \nu}$, and $\left(k_{A F}\right)_{\mu}$ are odd under CPT, while those with $H_{\mu \nu}, c_{\mu \nu}, d_{\mu \nu}$, and $\left(k_{F}\right)_{\kappa \lambda \mu \nu}$ preserve CPT. All ten terms break Lorentz symmetry. Typically, experiments can have different sensitivities to different types of Lorentz violation and can involves different particle species. For this reason, superscript labels are added to the SME coefficients in the fermion sector to denote the particle species. Lagrangian terms of the same form are expected to describe protons and neutrons in QED systems as well, but where the SME coefficients represent composites stemming from quark and gluon interactions.

\subsection{Extensions in Quantum Mechanics}

Many of the sharpest tests of Lorentz symmetry are conducted in high-precision particle and atomic experiments. Typically, static electric and magnetic fields are used in these experiments to trap or control charged particles, while the frequencies of particle transitions between different energy levels are measured with exceptional sensitivity. The electric and magnetic fields can also be manipulated to allow switching between particles and antiparticles, thereby permitting tests of CPT. The leading-order shifts in the standard (Lorentz- and CPT-preserving) energy levels are due to the effects of the small quantities $a_{\mu}, b_{\mu}, H_{\mu \nu}, c_{\mu \nu}, d_{\mu \nu}, e_{\mu}, f_{\mu}$, and $g_{\lambda \mu \nu}$. Lorentz violation stemming from couplings to the photon coefficients $\left(k_{A F}\right)_{\mu}$ and $\left(k_{F}\right)_{\kappa \lambda \mu \nu}$ enters only at subleading order for these types of measurements.

It is often sufficient in calculations describing these systems to work at the level of relativistic quantum mechanics using a modified Dirac equation. It is obeyed by a four-component spinor field $\psi$ describing a particle with charge $q$ and mass $m$. Calculation of leading-order energy shifts can be carried out most readily within a perturbative framework. To do so requires extracting a suitable Dirac hamiltonian from the lagrangian. However, the appearance of time-derivative couplings in the modified Dirac equation means that the standard procedure for obtaining the Dirac 
hamiltonian fails to produce a hermitian quantum-mechanical operator generating time translations on the wave function. This technical difficulty can be overcome by performing a field redefinition at the lagrangian level, chosen to eliminate the additional time derivatives. Rewriting the lagrangian in terms of the new field $\chi$ does not affect the physics. However, the modified Dirac wave function corresponding to $\chi$ does have conventional time evolution.

The rewritten Dirac equation takes the form

$$
i \partial_{0} \chi=\hat{H} \chi
$$

with

$$
\hat{H}=\hat{H}_{0}+\hat{H}_{\text {pert }} \text {. }
$$

In this notation, $\hat{H}_{0}$ is a conventional Dirac hamiltonian representing a charged particle in the absence of Lorentz- and CPT-violating perturbations. The perturbative hamiltonian $\hat{H}_{\text {pert }}$ for the particle is linear in the SME coefficients. The static electromagnetic fields enter in the perturbative treatment at leading order only through the dependence of the gauge-covariant derivatives on the background potential $A_{\mu}$.

In many experiments, energies are probed only at extremely low energy, where an expansion of the Hamiltonian in a nonrelativistic limit is appropriate. This can be implemented following a Foldy-Wouthuysen approach [51]. The resulting nonrelativistic perturbative hamiltonian can be written in terms of the three-momentum of the particle $p_{j}$ and the usual Pauli matrices $\sigma^{j}$ obeying $\left[\sigma^{j}, \sigma^{k}\right]=2 i \varepsilon_{j k l} \sigma^{l}$. The leading-order terms are

$$
\begin{aligned}
H_{\text {nonrel } \simeq} & m+\frac{p^{2}}{2 m} \\
& +\left(\left(a_{0}\right)-m c_{00}\right)+ \\
& +\left(-b_{j}+m d_{j 0} \frac{1}{2} \epsilon_{j k l} H_{k l}\right) \sigma^{j} \\
+ & {\left[-a_{j}+m\left(c_{o j}+c_{j o}\right)\right] \frac{p_{j}}{m}+\cdots . }
\end{aligned}
$$

In nonrelativistic experiments with ordinary matter the primary sensitivity will be to particular combinations of SME coefficients appearing in these terms. Subleading contributions can be calculated from expectation values of the terms involving factors of $p_{j}$, where the momentum is treated as a quantum-mechanical operator.

For experiments designed to test CPT, which involves measurements of both particles and antiparticles, the Dirac hamiltonian for the antiparticle must also be obtained. 


\begin{tabular}{|c|c|c|c|c|c|c|c|}
\hline SME Coeff. & C & P & T & CT & CP & TP & CPT \\
\hline \hline$a_{0}$ & - & + & + & - & - & + & - \\
\hline$a_{j}$ & - & - & - & + & + & + & - \\
\hline$b_{0}$ & + & - & + & + & - & - & - \\
\hline$b_{j}$ & + & + & - & - & + & - & - \\
\hline$H_{0 j}$ & - & - & + & - & + & - & + \\
\hline$H_{j k}$ & - & + & - & + & - & - & + \\
\hline$c_{00}$ & + & + & + & + & + & + & + \\
\hline$c_{0 j}$ & + & - & - & - & - & + & + \\
\hline$c_{j 0}$ & + & - & - & - & - & + & + \\
\hline$c_{j k}$ & + & + & + & + & + & + & + \\
\hline$d_{00}$ & - & - & + & - & + & - & + \\
\hline$d_{0 j}$ & - & + & - & + & - & - & + \\
\hline$d_{j 0}$ & - & + & - & + & - & - & + \\
\hline$d_{j k}$ & - & - & + & - & + & - & + \\
\hline
\end{tabular}

Table 1: Transformation properties of dominant SME terms in the matter QED limit under the discrete symmetries C,P,T and their combiinations.

This is accomplished using charge conjugation. The modified Dirac equation for the antiparticle differs from that of the particle by the sign of the charge $q$ and in the sign of any SME coefficients that are odd under charge conjugation. See Table 1 for a list of transformation properties for some of the dominant terms in the QED limit of the mSME.

All of the expressions in the quantum-mechanical limits depend explicitly on the spatial components $j, k, l$ of the SME coefficients and on the components of various physical quantities, such as the the particle momenta and the potential $A_{\mu}$. These components are defined with respect to a laboratory frame that must be chosen with a particular orientation. In laboratory frames fixed with respect to the surface of the Earth, the $j=3$ (or $z$ direction) is usually chosen as the relevant quantization axis, typically corresponding to the direction of a static magnetic field. Alternatively, if a rotation device is used on Earth's surface, such as a turntable, its orientation can be chosen as the $j=3$ direction. In a moving lab, such as in a satellite orbiting the Earth, a standard configuration defines the $j=3$ direction along the satellite velocity with respect to Earth, with the $j=1$ direction pointing toward Earth and 
the $j=2$ direction completing the right-handed system. In certain situations, Earthbased experiments may choose to use a satellite-based configuration as well, where the velocity of motion is due to the rotation of the Earth about its axis. The objective in this case is to take boost effects into account on the surface of the Earth as is done in satellite experiments. Ultimately, no matter which of these alignments is chosen for the lab frame directions labeled by $j$, the laboratory axes must be referenced to a nonrotating basis that can serve as a standard, since it is only with such a standard basis that comparisons across different experiments can be made. Bounds on components of SME coefficients in the lab frame must therefore be mapped into bounds on their components with respect to the standard reference frame.

For the standard reference frame, there are a number of different choices that could be made. Examples include reference frames attached to the centers of mass of the Earth, the Sun, the Milky Way galaxy, and the cosmic microwave background radiation (CMBR). With the exception of the Earth, each is approximately inertial over thousands of years. Typically in experiments, a Sun-centered celestial equatorial frame is chosen as the standard reference frame. It is used as the basis for reporting sensitivities to Lorentz violation. In certain limits, e.g., over short time scales where effects of boosts can be ignored, the spatial Sun-centered spatial components reduce to corresponding values in an Earth-based frame. Similarly, observer transformations from the Sun frame to a galaxy-based or CMBR-based frame can be made if bounds are desired with respect to these frames.

\subsection{Gravity Sector}

The gravity sector of the SME uses a vierbein formalism, which gives the theory a close parallel to gauge theory. Lorentz breaking occurs due to the presence of SME coefficients, which remain fixed under particle Lorentz transformations in a local frame. In this case, the SME coefficients carry Latin indices, e.g., $b_{a}$ for a vector, with respect to the local basis set. The conversion to spacetime coordinates is implemented by the vierbein, giving, e.g., $b_{\mu}=e_{\mu}{ }^{a} b_{a}$. The lagrangian can then be written in terms of fields and SME coefficients defined on the spacetime manifold. A natural (though not required) assumption is that the SME coefficients are smooth functions over the manifold. It is not necessary to require that they be covariantly constant. In fact, defining covariantly constant tensors over a manifold places stringent topological constraints on the geometry. One simplifying assumption, which could occur naturally in the context of spontaneous Lorentz breaking, is to assume that the SME coefficients 
are constants in the local frame. However, again, this is not a requirement in the formulation of the SME theory.

To construct the minimal SME including gravity [5], the first step is to incorporate gravitational fields into the usual SM. This is done by rewriting all of the terms in the SM Lagrangian with fields and gamma matrices defined with respect to the local frame (using Latin indices). The vierbein is then used to convert these terms over to the spacetime manifold. Factors of the determinant of the vierbein $e$ are included as well so that integration of the lagrangian density (giving the action) is covariant. Derivatives are understood as well to be both spacetime and gauge covariant. With these changes, Eq. (16), for example, becomes

$$
\begin{aligned}
\mathcal{L}_{\text {lepton }}= & \frac{1}{2} \operatorname{iee}_{a}^{\mu} \bar{L}_{A} \gamma^{a} \stackrel{\leftrightarrow}{D}_{\mu} L_{A} \\
& +\frac{1}{2} \operatorname{iee}_{a}{ }_{a} \bar{R}_{A} \gamma^{a} \stackrel{\leftrightarrow}{D}_{\mu} R_{A} .
\end{aligned}
$$

The other terms for the quark, Yukawa, Higgs, and gauge sectors follow a similar pattern.

The Lorentz-violating SME terms constructed from SM fields are obtained in a similar way. The various particle sectors can again be divided between CPT odd and even contributions. Each of the terms in the Lagrangian is then written using local indices and vierbeins, which convert the equations over to the spacetime manifold. As an example, Eq. (18) becomes

$$
\begin{aligned}
\mathcal{L}_{\text {lepton }}^{\mathrm{CPT}-\text { even }}= & -\frac{1}{2} i\left(c_{L}\right)_{\mu \nu A B} e e^{\mu}{ }_{a} \bar{L}_{A} \gamma^{a} \stackrel{\leftrightarrow}{D^{\nu}} L_{B} \\
& -\frac{1}{2} i\left(c_{R}\right)_{\mu \nu A B} e e^{\mu}{ }_{a} \bar{R}_{A} \gamma^{a} \stackrel{\leftrightarrow}{D^{\nu}} R_{B} .
\end{aligned}
$$

The remaining equations follow the same pattern.

The pure-gravity sector of the minimal SME consists of a Lorentz-invariant gravity sector and a Lorentz-violating sector. The Lorentz-invariant lagrangian consists of terms that are products of the gravitational fields. In the general case, this includes terms constructed from curvature, torsion, and covariant derivatives. Einstein's gravity (with or without a cosmological term) would be a special case in this sector.

The Lorentz-violating lagrangian terms in the gravity sector of the minimal SME are constructed by combining the SME coefficients with gravitational field operators to 
produce an observer scalar under local Lorentz transformations and general coordinate transformations. These consist of products of the vierbein, the spin connection, and their derivatives, but for simplicity they can be written in terms of the curvature, the torsion $T_{\lambda \mu \nu}$, and covariant derivatives. A minimal case (up to dimension four) has the form:

$$
\begin{aligned}
\mathcal{L}_{e, \omega}^{\mathrm{LV}}= & e\left(k_{T}\right)^{\lambda \mu \nu} T_{\lambda \mu \nu}+e\left(k_{R}\right)^{\kappa \lambda \mu \nu} R_{\kappa \lambda \mu \nu} \\
& +e\left(k_{T T}\right)^{\alpha \beta \gamma \lambda \mu \nu} T_{\alpha \beta \gamma} T_{\lambda \mu \nu} \\
& +e\left(k_{D T}\right)^{\kappa \lambda \mu \nu} D_{\kappa} T_{\lambda \mu \nu} .
\end{aligned}
$$

The SME coefficients in this expression have the symmetries of the associated Lorentzviolating operators that they multiply.

The Lorentz-violating sector introduces additional gravitational couplings that can have phenomenological consequences, including effects on cosmology, black holes, gravitational radiation, and post-Newtonian physics. As a starting point for a phenomenological investigation of the gravitational consequences of Lorentz violation, it is useful to write down the Riemannian limit of the minimal SME gravity sector. It is given as [5]

$$
\begin{aligned}
S_{e, \omega, \Lambda}= & \frac{1}{2 \kappa} \int d^{4} x[e(1-u) R-2 e \Lambda \\
& \left.+e s^{\mu \nu} R_{\mu \nu}+e t^{\kappa \lambda \mu \nu} R_{\kappa \lambda \mu \nu}\right] .
\end{aligned}
$$

The SME coefficient $\left(k_{R}\right)^{\kappa \lambda \mu \nu}$ has been expanded into coefficients $s^{\mu \nu}, t^{\kappa \lambda \mu \nu}, u$ that distinguish the effects involving the Riemann, Ricci, and scalar curvatures. The coefficients $s^{\mu \nu}$ have the symmetries of the Ricci tensor, while $t^{\kappa \lambda \mu \nu}$ has those of the Riemann tensor. Taking tracelessness conditions into account, there are 19 independent components.

\subsection{Spontaneous Lorentz Violation}

There are a number of theoretical issues concerning Lorentz violation that can be examined using the SME. One concerns the nature of the symmetry breaking and how that affects the interpretation of the SME coefficients. These coefficients, e.g., $b_{\mu}$, for the case of a vector, couple to SM and gravitational fields as fixed backgrounds. For the case of a single fermion field, $\psi$, in special relativity, the coupling has the form, 
$b_{\mu} \bar{\psi} \gamma^{5} \gamma^{\mu} \psi$. If this is the only term in the SME Lagrangian containing the coefficient $b_{\mu}$, then the symmetry breaking is said to be explicit. Essentially the coefficient $b_{\mu}$ appears in the effective field theory without any underlying dynamics. However, it is also possible for the SME coefficients to arise through a process of spontaneous symmetry breaking. In this case, the SME coefficients are interpreted as vacuum expectation values (or vevs) of a dynamical tensor field. For example, for a vector $B_{\mu}$, the SME coefficient would arise as a vev, $\left\langle B_{\mu}\right\rangle=b_{\mu}$. The vev acts as a fixed background field that spontaneously breaks Lorentz symmetry, but the vector $B_{\mu}$ remains fully dynamical.

The process of spontaneous symmetry breaking is important in particle physics. For example, in the electroweak theory, the scalar Higgs field $\phi$ acquires a nonzero vev, $\langle\phi\rangle \neq 0$, that spontaneously breaks the local $S U(2) \times U(1)$ gauge symmetry. For a scalar field, there is no associated breaking of Lorentz symmetry because the scalar vev is invariant under Lorentz transformations. However, the SME coefficients have tensor indices. When these occur as nonzero vevs, then Lorentz symmetry is said to be spontaneously broken.

The standard construction of the SME does not make a distinction between whether the breaking of Lorentz symmetry is explicit or spontaneous. Both types of symmetry breaking can be accommodated, and both are useful to consider for phenomenological investigations of Lorentz violation. However, when the gravitational sector of the SME is included, which brings more geometrical considerations into play, it becomes important to distinguish these types of symmetry breaking.

For the case of explicit Lorentz violation, it has been shown that inconsistencies arise between geometrical constraints (e.g., Bianchi identities) and conditions stemming from the equations of motion. This was proved by Kostelecký in a no-go theorem [5]. However, the no-go theorem is evaded if the symmetry breaking is spontaneous. The crux of the difference has to do with the fact that if the Lorentz breaking is spontaneous, then all of the SME coefficients have to be treated as dynamical fields in field variations.

Because of this, it is often assumed that the SME coefficients are indeed vevs of dynamical fields that have undergone a process of spontaneous Lorentz breaking. Note, however, that if the vevs are associated with very high energy scales, then in low-energy tests of Lorentz violation, they will still act primarily as fixed background fields, and their dynamics at higher energies will not be relevant. It is for this reason that the form of the SME or mSME used by most experimentalists is the same as if 
the symmetry breaking were explicit. For purposes of phenomenology, the distinction between explicit and spontaneous Lorentz breaking is not crucial. For the case of explicit breaking, it may be that a different type of geometry is relevant, known as a Riemann-Finsler geometry. (For a review, see [52]). The SME with explicit breaking has been shown to be linked to Riemann-Finsler geometry [53, 54].

It is certainly the case that spontaneous symmetry breaking is a very elegant form of symmetry breaking. This is because when a symmetry is spontaneously broken, the symmetry still holds dynamically. However, the vacuum solution for the theory does not obey the symmetry. What is often done is that a field redefinition is performed that resets the vacuum values to zero. In this case, in terms of the new set of fields, the symmetry becomes hidden at the level of the equations of motion. It is for this reason that spontaneous symmetry breaking is also referred to as hidden symmetry.

From a theoretical point of view, there are well-known consequences when a symmetry is spontaneously broken. For example, when a global continuous symmetry is spontaneously broken, it has been shown that massless fields, called Nambu-Goldstone (NG) fields appear $[55,56,57]$. On the other hand, if the symmetry is local, as in the case of the electroweak model, then a Higgs mechanism can occur $[58,59,60]$. In this case, the would-be NG modes get reinterpreted in a way that results in the gauge fields acquiring a mass. This is what happens in the electroweak model, and as a result the $\mathrm{W}$ and $\mathrm{Z}$ bosons are massive. However, an unbroken local $\mathrm{U}(1)$ gauge symmetry allows the photons to remain massless. At the same time, there are excitations of the Higgs scalar field that are also massive. This results in a massive Higgs boson, which has recently been detected at the Large-Hadron Collider.

An important theoretical issue to consider is whether these same types of processes can occur when it is Lorentz symmetry that is spontaneously broken. For the case where Lorentz symmetry is global, as in the context of special relativity, the Goldstone theorem would suggest that massless NG modes should appear. If so, they would appear as infinite-range particles and would have implications for phenomenology. The only known massless particles in the SM and GR (assuming neutrinos have mass) are the gauge fields, such as the photon, graviton, and gluons. Thus, it would seem that there are only two possibilities for the NG modes. Either the NG modes are known particles, such as photons or gravitons, or they are unknown fields that have escaped detection. However, if the Lorentz symmetry is local, as in a gravitational theory, then the question of whether a Higgs mechanism can occur becomes relevant. In this case, the possibility of massive gauge fields arises (massive photons or massive 
gravity), and the question of whether there are additional massive Higgs fields needs to be addressed as well.

These types of questions have been investigated both in special relativity and in the context of gravity using models that are subsets of the SME. Interestingly, some of these investigations occurred before the process of spontaneous symmetry breaking was fully understood. For example, Dirac worked with a vector model that had a constraint that the norm of the vector be nonzero [61]. Nambu later showed that such a model spontaneously breaks Lorentz symmetry [62]. Bjorken found a similar model using a composite theory of fermions that collectively have a nonzero vector vev [63]. It was conjectured that in these types of models, the NG modes can be interpreted as photons. This raises the interesting possibility that photons are massless because they are the NG modes associated with spontaneous Lorentz breaking, whereas the conventional idea is that photons are massless because of local gauge invariance.

In order to impose a constraint that a vector field has a nonzero vev, the usual process in field theory is to include a potential term that has a minimum when the vector field equals its vev. Theories with a vector field and a potential of this type that induces spontaneous Lorentz violation are known as bumblebee models $[5,15,64,65,66,67,68,69,71,70,72,73,74,75,76]$. A defining feature of these theories is that they do not have local $\mathrm{U}(1)$ gauge invariance. Thus, there is no possibility in these models for photons to arise because of local U(1) gauge symmetry. Recent investigations of bumblebee models have shown that all of the usual processes associated with spontaneous symmetry breaking can occur when the symmetry is Lorentz symmetry. First, however, it was found that there is a link between local Lorentz symmetry and diffeomorphisms. In general, if one of these symmetries is spontaneously broken, then so is the other. For example, if a vector field has a vev $b_{a}$ in a local Lorentz frame, which spontaneously breaks LLI, then it will also have a vev $b_{\mu}$ in the spacetime frame, which spontaneously breaks diffeomorphisms. (Even for a scalar vev with spacetime dependence this is true, though in this case it is the derivatives of the scalar that spontaneously break the symmetries). What this means is that in the context of a gravitational theory with spontaneous Lorentz breaking there can be up to ten NG modes, six associated with Lorentz breaking, and four associated with diffeomorphism breaking.

If these symmetries are treated analogously to local gauge symmetry using a vierbein formalism, then it is possible to show that the vierbein itself can accommodate all 
ten NG modes when local Lorentz symmetry and diffeomorphisms are spontaneously broken. It is also possible to investigate whether a Higgs mechanism can occur and whether additional massive Higgs modes can appear. Interestingly, it is found that for a Higgs mechanism to occur the geometry cannot be Riemannian. This is because the gauge fields associated with the local Lorentz symmetry are the spin connection, and in order to have a dynamical spin connection, the theory must include torsion. The geometry must therefore be Riemann-Cartan if a Higgs mechanism is to occur. There can also be additional massive Higgs modes that can affect the propagation of metric excitations (or gravitational radiation). It is for this reason that theories of massive gravity often result from the process of spontaneous Lorentz violation. In all of these models, there are stringent conditions that must hold so that unphysical modes do not appear, such as negative energy states or tachyons. These constraints very severely limit the possibilities for making viable models with massive gravitational fields or massive propagating spin connection.

A subset of the bumblebee models in which the kinetic term for the vector field has a Maxwell form, are known as Kostelecký-Samuel (KS) models [15]. For these models, it has been shown that in the limit where the massive Higgs modes becomes extremely massive, the solutions for the KS model match those of Einstein-Maxwell theory in a fixed gauge. Thus, the intriguing idea that photons might arise as NG modes in a theory with spontaneous Lorentz breaking still holds even when gravity is included.

It is also possible to consider models with other types of tensor fields that acquire nonzero vacuum values. Some possibilities include theories with a symmetric twotensor or alternatively an anti-symmetric two-tensor [77, 78, 79]. Just as with a vector, when Lorentz symmetry in these models is spontaneously broken, NG modes and massive modes can appear. It is useful to study these models to see what the various possibilities are for the NG and massive modes. One interesting case is that of a symmetric two-tensor in a Minkowski background. In this type of model, known as a cardinal model, the NG modes have properties similar to the graviton in GR, but in a fixed gauge. This again raises the intriguing question of whether known massless particles might occur as a result of Lorentz breaking. A related consideration is then whether there exist signatures of the Lorentz breaking that can distinguish KS and cardinal models from conventional physics. These types of phenomenological questions can then be suitably addressed in the context of the SME.

In cosmology, models with spontaneous Lorentz violation have been used to study modifications of gravity that might give rise to effects such as accelerated expan- 
sion of the universe or to introduce anisotropic features in the cosmic background radiation. Examples include $[80,81,82,83,84,85,86]$. In general, these models, which incorporate vector or tensor fields that spontaneously break Lorentz symmetry, are studied as possible alternative theories of dark energy. While these theories have a number of interesting effects and features, they do not typically give rise to high-precision observational constraints on LLI. For this reason, these models are not considered here, and the reader is referred to the literature.

\section{Experimental Tests of Lorentz Violation}

If Lorentz invariance is not an exact symmetry due to mechanisms occurring in the context of a quantum theory of gravity, then the relevant energy scale is presumably the Planck scale, since this is the scale where gravity meets up with quantum physics. At one time, it was thought unlikely that any physics arising from the Planck scale would be accessible to experimental detection. However, with Lorentz violation, the Planck scale is expected to enter as a suppression factor or inverse power in any corrections to conventional physics. Therefore, instead of needing to accelerate particles to ultra-high energies that are impossible to obtain, one can look at extremely highprecision experiments often at very low energies for signs of Planck-scale physics. In this approach, Lorentz breaking provides an ideal signal of new physics, since nothing in the SM permits violation of Einstein's theory. That is, no conventional process could ever mimic or cover up a genuine signal of Lorentz violation.

The SME serves as a common framework used by experimentalists and theorists to search for signals of Lorentz and CPT violation. Planck-scale sensitivity has been attained to the dominant SME coefficients in a number of experiments involving different particle sectors. These include experiments with mesons, photons, electrons, protons, neutrons, muons, neutrinos, and the Higgs. Each particle sector has unique features, and the experimental methods for testing Lorentz and CPT violation can differ case by case.

In some experiments, leading-order sensitivity to Lorentz and CPT violation exists for more than one particle species at the same time. This is particularly true in atomic experiments where bounds involving all three of the electron, proton, and neutron are often obtained. Likewise, mixtures of flavors in the meson and neutrino sectors can occur naturally. In these cases, the experimental bounds obtained are for combi- 
nations of SME coefficients for the different particle sectors. It is therefore important to look for complementary sets of bounds obtained from different experiments that can be combined to select out an optimal set of bounds for the individual particle species.

In a similar manner, experiments can have sensitivity to either both CPT-odd and CPT-even forms of Lorentz violation, or alternatively they can probe only the CPTodd sector. Most bounds obtained typically involve combinations of both CPT-odd and CPT-even SME coefficients. However, experiments designed to test CPT switch between measurements on particles and similar measurements on the corresponding antiparticles. The bounds in this case are only on CPT-odd SME coefficients. For a given particle species, performing both types of experiments provides a natural complementary approach.

Before looking at specific experiments, it is useful to examine some general features that are common to a number of different experiments. For example, in low-energy atomic tests, the sensitivity stems primarily from the ability of these experiments to detect extremely small anomalous energy shifts. In many cases, these energy shifts result in small frequency shifts that can be measured with very high precision. It is not uncommon for an atomic experiment to be able to measure a frequency shift with a precision of $1 \mathrm{mHz}$ or less. Interpreting this as being due to an energy shift expressed in $\mathrm{GeV}$, it corresponds to a sensitivity of approximately $4 \times 10^{-27} \mathrm{GeV}$. Such a value is well within the range of energy one might associate with suppression factors originating from the Planck scale. While many of the original atomic experiments were designed to measure specific quantities, such as charge-to-mass ratios of particles and antiparticles or differences in $g$ factors, it turns out that it is more effective for these experiments to investigate the lowest attainable energy levels for possible anomalous shifts associated with Lorentz violation. Many experiments look specifically for sidereal time variations of energy levels of a particle or atom as the Earth moves. These would result from interactions with the fixed Lorentz-violating background fields. Alternatively, experiments designed to test CPT can look for instantaneous differences in the energy levels of a particle (or atom) and its antiparticle (or antiatom).

Another important general consideration is the choice of a standard inertial reference frame [87]. Laboratory measurements of Lorentz and CPT symmetry involve components of SME coefficients defined with respect to a local laboratory coordinate system. These components labeled with indices $\{0, j\}$ change as the lab frame moves 
or rotates with respect to an inertial frame. In order to give measured bounds in a consistent manner, these laboratory bounds must be related to bounds on SME coefficients defined with respect to a standard inertial frame. The usual choice for this frame is a Sun-centered frame that uses celestial equatorial coordinates. Components with respect to the Sun-centered frame are denoted using upper-case letters $J, K, L, \ldots$ that run over four independent directions labeled as $\hat{T}, \hat{X}, \hat{Y}, \hat{Z}$. The spatial origin of this system is the Sun's center, and the unit vector $\hat{Z}$ points along the Earth's rotation axis, while $\hat{X}$ and $\hat{Y}$ lie in the equatorial plane with $\hat{X}$ pointing towards the vernal equinox in the celestial sphere. The time $T$ is measured by a stationary clock at the origin, with $T=0$ taken as the vernal equinox in the year 2000 . The Earth's orbital plane lies at an angle $\eta \simeq 23^{\circ}$ with respect to the $X Y$ plane.

Earth-based experiments sensitive to sidereal time variations are sensitive to a combination of coefficients, which are often denoted collectively using tildes. For example, for electrons, the combination of spatial components in the lab frame

$$
\tilde{b}_{j}^{e} \equiv b_{j}^{e}-m d_{j 0}^{e}-\frac{1}{2} \varepsilon_{j k l} H_{k l}^{e}
$$

arises frequently in a number of experiments. These combinations are projected onto the nonrotating frame, where the components are $b_{X}^{e}, b_{Y}^{e}, b_{Z}^{e}$, etc. Nonrotating frame analogues of the coefficient combinations in 31 can be defined as

$$
\tilde{b}_{J}^{e} \equiv b_{J}^{e}-m d_{J 0}^{e}-\frac{1}{2} \varepsilon_{J K L} H_{K L}^{e},
$$

where $J, K, L$ label the spatial directions $X, Y, Z$ in the nonrotating frame. Ignoring boost effects, the relation between the laboratory and nonrotating spatial components is

$$
\begin{aligned}
\tilde{b}_{1}^{e}= & \tilde{b}_{X}^{e} \cos \chi \cos \Omega t \\
& +\tilde{b}_{Y}^{e} \cos \chi \sin \Omega t-\tilde{b}_{Z}^{e} \sin \chi, \\
\tilde{b}_{2}^{e}= & -\tilde{b}_{X}^{e} \sin \Omega t+\tilde{b}_{Y}^{e} \cos \Omega t, \\
\tilde{b}_{3}^{e}= & \tilde{b}_{X}^{e} \sin \chi \cos \Omega t \\
& +\tilde{b}_{Y}^{e} \sin \chi \sin \Omega t+\tilde{b}_{Z}^{e} \cos \chi .
\end{aligned}
$$

The angle $\chi$ is between the $j=3$ lab axis and the direction of the Earth's rotation axis along $Z$. The angular frequency $\Omega \simeq 2 \pi /(23 \mathrm{~h} 56 \mathrm{~m})$ is that corresponding to a sidereal day. 


\subsection{Data Tables}

A wide range of particle sectors has been investigated for Lorentz and CPT violation. Many experiments achieve very high sensitivity to Lorentz violation and are able to place stringent bounds on the relevant SME coefficients. The results for these bounds are too extensive to list here. However, a comprehensive summary of Lorentz and CPT tests has been published by Kostelecký's group at Indiana University [88]. It is also updated annually on the physics archive.

The data tables in Ref. [88] provide bounds on Lorentz violation for ordinary matter (electrons, protons, and neutrons), photons, mesons, muons, neutrinos, the Higgs, and gravity. Many tests compare particles and anti-particles. Low-energy tests in atomic physics include experiments in Penning traps, comparisons of atomic clocks and masers, experiments with atomic fountains, and experiments with antihydrogen at CERN. Photon tests have been performed using astrophysical and cosmological sources as well as resonant cavities in the microwave and optical regimes. Cosmic rays have been investigated for features associated with Lorentz violation. Experiments with mesons, muons, and neutrinos have used large accelerators at high energies. Experiments are planned or underway on the International Space station, in space satellites, or using detectors at the south pole. Experiments with macroscopic torsion pendula take advantage of the alignment of large numbers of electron spins to provide bounds with extremely high sensitivity. To measure boost effects, some experiments collect data over long periods of time to enable the Earth's motion to be included. Other experiments use rotating platforms to gain sensitivity to a wider range of space-time directions.

The extremely tight experimental bounds that have been obtained on the leadingorder SME coefficients indicate that if Lorentz or CPT violation does occur in nature, it results in only very small corrections to the SM and GR at ordinary energies. Since an underlying fundamental theory that would permit calculation of these corrections is lacking, at best only order-of-magnitude estimates can be given for the leadingorder SME coefficients. One possibility is that the leading-order Lorentz-violating terms in the SME are suppressed by at least one inverse power of the Planck scale. If a ratio is formed with a low-energy scale on the order of $1 \mathrm{GeV}$ with the Planck scale, this results in a suppression factor on the order of $10^{-19}$. Interestingly, many of the recent experiments that test Lorentz and CPT symmetry have sensitivities that are comparable to or exceed expected order-of-magnitude values based on this suppression factor. For this reason, it is important as well to search for Lorentz violation 
stemming from sub-leading-order terms that are not included in the mSME. A systematic treatment of these higher-dimensional terms in the SME has been developed for certain particle sectors, and bounds on some of these coefficients are included in the data tables as well.

\subsection{Examples}

To highlight some of the Lorentz and CPT tests that have been performed a number of different experimental approaches are described here. In many cases, bounds on a selective subset of SME coefficients are given. For a full list of experiments with published bounds on SME coefficients, the reader is referred to the data tables in [88].

- Penning Traps [89, 90, 91, 92, 93]: Experiments in Penning traps use electric and magnetic fields to isolate and study individual particles and antiparticles. There are two leading-order signals of Lorentz and CPT violation in the electron sector that have been probed in these experiments. One looks for sidereal time variations in the electron cyclotron and anomaly frequencies. The idea here is that the Lorentz and CPT-violating interactions depend on the orientation of the quantization axis in the laboratory frame, which changes as the Earth turns on its axis. As a result, both the cyclotron and anomaly frequencies have small corrections which cause them to exhibit sidereal time variations. Such a signal can be measured using just electrons. Measured bounds are expressed in terms of components in the nonrotating Suncentered frame for the combination given in Eq. 32. Their numerical values are on the order of $\left|\tilde{b}_{J}^{e}\right| \lesssim 10^{-24} \mathrm{GeV}$ for $J=X, Y$. The second type of test in a Penning trap is a traditional CPT test that compares electrons and positrons directly. It looks for an instantaneous difference in their anomaly frequencies. Leading-order sensitivity in this case involves only the CPT-odd coefficient $b_{3}^{e}$ (with no tilde), which is the component of $b_{\mu}^{e}$ along the quantization axis in the laboratory frame. The bound obtained for $\left|b_{3}^{e}\right|$ is on the order of $10^{-25} \mathrm{GeV}$.

- Torsion Pendulum [94, 95, 96, 97]: Experiments using a spin-polarized torsion pendulum are able to achieve very high sensitivity to Lorentz violation because the torsion pendulum has a huge number of aligned electron spins but a negligible magnetic field. For example, a pendulum at the University of Washington is built out of a stack of toroidal magnets, which has a net electron spin $S \simeq 10^{23}$. The apparatus is suspended on a rotating turntable and the time variations of the twisting pendulum are mea- 
sured. An analysis of this system shows that in addition to a signal having the period of the rotating turntable, the effects due to Lorentz and CPT violation also cause additional time variations with a sidereal period caused by the rotation of the Earth. Sensitivity to the electron coefficients has been obtained at the levels of $\left|\tilde{b}_{J}^{e}\right| \lesssim 10^{-31}$ $\mathrm{GeV}$ for $J=X, Y$ and $\left|\tilde{b}_{Z}^{e}\right| \lesssim 10^{-30} \mathrm{GeV}$. By analyzing data over the course of a year, taking the Earth's motion around the Sun into account, sensitivity to Lorentzboost violating coefficients has been attained as well. This involves a suppression by $v / c \simeq 10^{-4}$, where $v$ is the velocity of the Earth around the Sun. The bound on the timelike combination of coefficients is $\tilde{b}_{T}^{e} \lesssim 10^{-27} \mathrm{GeV}$.

-Clock-Comparison Tests [87, 98, 99, 100, 101, 102, 103, 105, 106, 107]: Many of the sharpest Lorentz bounds for the proton and neutron stem from atomic clockcomparison experiments. These involve making high-precision comparisons of atomic clock signals as the Earth rotates. The clock frequencies are typically hyperfine or Zeeman transitions. Experiments have used hydrogen masers and two-species noblegas masers to achieve the highest sensitivities to Lorentz violation. For example, a recent experiment with a $\mathrm{K}-\mathrm{He}^{3}$ co-magnetometer obtained a bound in the neutron sector equal to $\left|\tilde{b}_{J}^{n}\right| \lesssim 10^{-33} \mathrm{GeV}$ for $J=X, Y$. Experiments with hydrogen masers attain exceptionally sharp sensitivity to Lorentz and CPT violation in the electron and proton sectors. These experiments use a double-resonance technique that does not depend on there being a field-independent point for the transition. The sensitivity for the proton attained in these experiments is $\left|\tilde{b}_{J}^{p}\right| \lesssim 10^{-27} \mathrm{GeV}$. Due to the simplicity of hydrogen, this is an extremely clean bound and is one of the more stringent tests for the proton. Clock-comparison experiments performed in space would have several advantages over traditional ground-based experiments. For example, a clock-comparison experiment conducted aboard the International Space Station (ISS) would be in a laboratory frame that is both rotating and boosted. It would therefore immediately gain sensitivity to a wide range of SME coefficients that are currently untested [108, 109]. A European mission is planned for the ISS which will compare atomic clocks and $\mathrm{H}$ masers.

- Antihydrogen [98, 110, 111]: The ALPHA and ATRAP experiments underway at CERN are designed to produce antihydrogen and to do high-precision spectroscopy on it. One objective is to make high-precision spectroscopic measurements of the $1 \mathrm{~S}-2 \mathrm{~S}$ transitions in hydrogen and antihydrogen. These are forbidden (two-photon) transitions that have a relative linewidth of approximately $10^{-15}$. The ultimate goal is to measure the line center of this transition to a part in $10^{3}$ yielding a frequency comparison between hydrogen and antihydrogen at a level of $10^{-18}$. An alternative 
to $1 \mathrm{~S}-2 \mathrm{~S}$ transitions is to consider the sensitivity to Lorentz violation in ground-state Zeeman hyperfine transitions. It is found that there are leading-order corrections in these levels in both hydrogen and antihydrogen. Comparing these measurements for hydrogen and antihydrogen will provide a direct CPT test.

-Photon Tests $[112,113,114,115,116,117,118,119,120,121,122,123,124]$ : The relevant leading-order terms for the photon sector in the SME are the $k_{A F}$ and $k_{F}$ terms in Eq. (23). For the coefficient $k_{A F}$, which is odd under CPT, it is found theoretically that this term leads to negative-energy contributions and is a potential source of instability in the theory unless it is set to zero [125]. In addition, very stringent experimental constraints that come from studying the polarization of radiation from distant radio galaxies also exist and are consistent with $k_{A F} \approx 0$. The terms with coefficients $k_{F}$ are even under CPT and provide positive-energy contributions. There are 19 independent components in the $k_{F}$ coefficients. Ten of them lead to birefrigence of light. Bounds on these coefficients of order $10^{-32}$ have been obtained from spectropolarimetry of light from distant galaxies. The remaining nine coefficients have been bounded in a series of laboratory photon experiments. These include experiments using optical and microwave cavities, an Ives-Stilwell experiment, and experiments using rotating platforms. Sensitivities ranging from $10^{-9}$ up to $10^{-17}$ have been attained for these coefficients.

- Cosmic Rays [126, 127, 128]: Cosmic rays provide the highest-energy particles available experimentally and can be used to study LLI. In the presence of Lorentz violation, the maximal attainable velocity for a cosmic ray in vacuum can be different from the speed of light by a small amount. In principle, it can even exceed the speed of light. Effects of this difference include the possibility of photon decay into electron-positron pairs or vacuum Cerenkov radiation by ultra-high-energy electrons, both of which are forbidden in the SM. Another effect is the prediction in the context of the SM and special relativity that an upper energy limit known as the Greisen - Zatsepin Kuzmin, or GZK limit [129, 130], should hold for cosmic rays emitted from distant sources. This theoretical limit is set by interactions with the cosmic microwave background radiation over long distances. However, in the presence of Lorentz violation, it is possible for high-energy cosmic rays from distant sources to exceed the GZK limit. This therefore provides an opportunity for testing LLI and obtaining bounds on the relevant SME coefficients. Recent experiments at the High Resolution Fly's Eye (HiRes) and Pierre Auger Observatory have searched for ultra-high-energy cosmic rays above the GZK limit, and their results appear to confirm the existence of the GZK cutoff. 
- Meson Tests [131, 132, 133, 134, 135, 136, 137]: Experiments involving neutral meson oscillations provide very sharp tests of Lorentz and CPT symmetry. These investigations attain high sensitivity to the CPT-odd $a_{\mu}$ coefficients in the SME for the $K, D, B_{d}$, and $B_{s}$ meson systems. The time evolution of a meson and its antimeson can be described by an effective hamiltonian in a description based on the Schrödinger equation. The dominant Lorentz- and CPT-violating contributions to the effective hamiltonian can be calculated as expectation values of interaction terms in the SME. The results depend on the velocity of the meson with respect to the laboratory frame and the combinations of SME coefficients $\Delta a_{\mu}$, which vary with sidereal time as the Earth rotates. Recent analyses have attained bounds on the order of $10^{-21} \mathrm{GeV}$ for neutral kaons, $10^{-15} \mathrm{GeV}$ in the $\mathrm{D}$ system, $10^{-14} \mathrm{GeV}$ for $B_{d}$ oscillations, and $10^{-12}$ $\mathrm{GeV}$ for $B_{s}$ oscillations.

- Muon Tests [138, 139, 140]: Lorentz and CPT tests with muons involve secondgeneration leptons and are independent of the tests involving electrons. Several different types of experiments with muons have been conducted, including muonium experiments and $g-2$ experiments with muons. In muonium, experiments measuring the frequencies of ground-state Zeeman hyperfine transitions in a strong magnetic field have the greatest sensitivity to Lorentz and CPT violation. A recent analysis has searched for sidereal time variations in these transitions. A bound on SME coefficients, $\left|\tilde{b}_{J}^{\mu}\right|$, has been obtained at a level of $10^{-23} \mathrm{GeV}$. In relativistic $g-2$ experiments using positive and negative muons bounds on Lorentz-violation SME coefficients have been obtained at a level of $10^{-24} \mathrm{GeV}$.

- Collider Tests [141, 142, 143, 144]: High energy experiments at colliders provide opportunities for testing Lorentz and CPT violation in the QED and quark sectors. Sensitivity for Lorentz violation in cross sections and decay rates has been investigated in electron-positron scattering. Effects include variations in observed cross sections with periodicities controlled by Earth's sidereal rotation frequency. In a recent experiment using the D0 detector at the Fermilab Tevatron Collider, a search for violation of Lorentz invariance in the top quark-antiquark production cross section was carried out, and bounds on SME coefficients for the top quark were obtained.

- Neutrino Tests $[145,146,147,148,149,150,151,152,153,154,155]$ : The experimental observation that neutrinos change flavor when they propagate through space cannot be explained by the SM. The conventional explanation for these neutrino oscillations is that the particles have very small masses. However, at the same time, the high-precision sensitivity of neutrino oscillation experiments, stemming from their in- 
terferometric nature, offers possibilities for a range of new tests of LLI. The neutrino sector of the mSME contains Lorentz-violating interactions for left-handed neutrinos and right-handed antineutrinos. For the left-handed neutrinos, sensitivity at leading order is to the SME coefficients $\left(a_{L}\right)^{\mu}$ and $\left(c_{L}\right)^{\mu \nu}$. The resulting signals include ones with the usual $L / E$ dependence, where $E$ is the energy and $L$ is the oscillation length or baseline of the experiment. However, with Lorentz violation other dependences, such as ones with $L$ or $L E$ are possible as well. These lead to unique signatures of Lorentz violation that can occur in neutrino experiments. These include oscillation, time of flight, and threshold effects. For example, it has been shown that a Lorentz-violating seesaw mechanism can occur, which allows for oscillatory behavior even in the absence of mass. The coefficients for Lorentz violation can also couple to the four-momentum of the neutrino. In terrestrial experiments, the direction of the neutrino beam changes as the Earth rotates, which leads to sidereal time variations in the oscillation data when LLI is broken. The mSME has been applied to a number of neutrino experiments, including both short-baseline and long-baseline experiments. An extensive list of bounds on SME coefficients in the neutrino sector are given in the Data Tables [88]. For the coefficients $\left(a_{L}\right)^{\mu}$, bounds at the level of $10^{-20}$ to $10^{-23}$ $\mathrm{GeV}$ have been obtained, while for the $\left(c_{L}\right)^{\mu \nu}$ coefficients, the sensitivity ranges from $10^{-17}$ to $10^{-27}$.

- Gravity Tests [156, 157, 158, 159, 160, 161, 162, 163]: Lorentz violation in the gravity sector stems from both matter-gravity couplings and pure-gravity couplings. In some cases, the matter-gravity couplings can lead to sensitivity to forms of Lorentz violation that would otherwise go undetected in the absence of gravity. The leading-order SME terms for both these sectors in a linearized gravity regime involve expectation values denoted as $\bar{a}_{\mu}, \bar{c}_{\mu \nu}$ and $\bar{s}_{\mu \nu}$. At leading order, matter-gravity tests are sensitive to $\bar{a}_{\mu}$ and $\bar{c}_{\mu \nu}$, while pure-gravity tests are sensitive to $\bar{s}_{\mu \nu}$. The matter-gravity tests include gravimeter, atom interferometry, and weak equivalence principle experiments. Bounds on $\bar{a}_{\mu}$ have been obtained at levels of $10^{-6}$ to $10^{-11} \mathrm{GeV}$ and on $\bar{c}_{\mu \nu}$ at the levels of $10^{-6}$ to $10^{-8}$. Tests sensitive to the pure-gravity couplings include experiments with atom interferometers, torsion pendula, and lunar and satellite laser ranging experiments. Bounds on $\bar{s}_{\mu \nu}$ coefficients at levels of $10^{-6}$ to $10^{-9}$ have been obtained. In addition to these gravity tests, highly sensitive tests attempting to detect spacetime torsion can be achieved by searching for its couplings to fermions [164]. Bounds on torsion components down to levels of $10^{-31} \mathrm{GeV}$ have been obtained in this way. 


\section{$5 \quad$ Summary and Conclusions}

Interest in the idea of Lorentz violation has steadily increased over the past two decades. This is due to theoretical advances showing that Lorentz breaking can provide unique signals of Planck-scale physics and quantum-gravity effects as well as to experimental advances that have led to new high-precision tests of LLI. The development and use of the SME as the theoretical framework describing Lorentz violation in the context of field theory has led to a comprehensive and multi-disciplinary approach to testing LLI that spans most of the particle sectors in the SM.

The underlying premise of the SME is that field theory and the SM are correct descriptions of particle interactions at low energies. Therefore, any indications of Lorentz violation should show up as small corrections in the context of effective field theory. The SME is constructed as the most general effective field theory that incorporates Lorentz violation. It contains all known particle fields and gravitational interactions as well as all observer-independent terms that break LLI. As an incremental first step, the minimal SME and its QED limit, which maintain gauge invariance and power-counting renormalizability, were constructed in the 1990s. These have been used extensively to search for leading-order signals of Lorentz and CPT violation. More recently, a systematic approach to constructing the nonminimal sectors of the SME have been worked out for certain particle species, and experimental bounds of these terms are being obtained as well $[165,166]$.

As a comprehensive theoretical framework, the SME allows for investigations of theoretical issues related to the idea of Lorentz violation. Specifically, for the case of spontaneous Lorentz breaking, investigations of the fate of the Nambu-Goldstone modes and the possibility of Higgs masses and a Higgs mechanism have been carried out. It has been shown that spontaneous Lorentz violation is accompanied by spontaneous diffeomorphism breaking, and up to 10 Nambu-Goldstone modes can appear in principle. These modes can comprise 10 of the 16 degrees of freedom of the vierbein, which in a Lorentz-invariant theory are gauge degrees of freedom. The fate of the Nambu-Goldstone modes is found to depend on the type of spacetime geometry in the underlying theory. At leading order in Minkowski and Riemann spacetimes, it is found that the Nambu-Goldstone modes can propagate like photons in a fixed axial gauge. However, in Riemann-Cartan spacetimes, the possibility exists that the spin connection can absorb the Nambu-Goldstone modes in a gravitational version of the Higgs mechanism. In addition, the potential inducing spontaneous Lorentz violation can provide mass terms for the metric excitations. These features create new possi- 


\begin{tabular}{|c|c|c|c|}
\hline \hline Expt & Sector & Params $(J=X, Y)$ & Bound $(\mathrm{GeV})$ \\
\hline \hline Penning Trap & electron & $\tilde{b}_{J}^{e}$ & $10^{-24}$ \\
\hline K-He dual maser & neutron & $\tilde{b}_{J}^{n}$ & $10^{-33}$ \\
\hline H maser & proton & $\tilde{b}_{J}^{p}$ & $10^{-27}$ \\
\hline Muonium & muon & $\tilde{b}_{J}^{\mu}$ & $10^{-23}$ \\
\hline Spin Pendulum & electron & $\tilde{b}_{J}^{e}$ & $10^{-31}$ \\
\hline \hline
\end{tabular}

Table 2: Summary of leading-order bounds for the coefficient $\tilde{b}_{J}$.

bilities for constructing models with spontaneous Lorentz violation in the context of massive gravity.

The main application of the SME has been in phenomenological investigations of Lorentz and CPT symmetry. High precision tests have been performed in most of the primary particle sectors in the SM. These include experiments in QED and atomic systems, astrophysical tests, and laboratory tests at nuclear and particle facilities. The generality of the SME allows comparisons across different types of experiments involving the same particle species. These tests have greatly improved the sensitivity to which Lorentz and CPT symmetry is known to hold, though many particle sectors, particularly those beyond leading order, remain to be probed. As a comparison of some of the bounds obtained to date at leading order, a summary of some bounds on $\tilde{b}_{J}$ coefficients in the minimal SME is given in Table 2 . These bounds are within the range of sensitivity associated with suppression factors arising from the Planck scale. A more complete set of tables for the full SME is published in the Indiana University data tables [88]. 


\section{References}

[1] V.A. Kostelecký and R. Potting, Phys. Rev. D 51, 3923 (1995).

[2] D. Colladay and V.A. Kostelecký, Phys. Rev. D 55, 6760 (1997).

[3] D. Colladay and V.A. Kostelecký, Phys. Rev. D 58, 116002 (1998).

[4] V.A. Kostelecký and R. Lehnert, Phys. Rev. D 63, 065008 (2001).

[5] V.A. Kostelecký, Phys. Rev. D 69, 105009 (2004).

[6] J. Schwinger, Phys. Rev. 82 (1951) 914.

[7] J.S. Bell, Proc. Roy. Soc. (London) A 231 (1955) 479.

[8] W. Pauli, in W. Pauli, ed., Neils Bohr and the Development of Physics, McGrawHill, New York, 1955, p. 30.

[9] G. Lüders, Ann. Phys. (N.Y.) 2 (1957) 1.

[10] O.W. Greenberg, Phys. Rev. Lett. 89, 231602 (2002).

[11] F.W. Hehl et al., Rev. Mod. Phys. 48, 393 (1976).

[12] I.L. Shapiro, Phys. Rep. 357, 113 (2002).

[13] V.A. Kostelecký and S. Samuel, Phys. Rev. D 39, 683 (1989).

[14] V.A. Kostelecký and S. Samuel, Phys. Rev. Lett. 63, 224 (1989).

[15] V.A. Kostelecký and S. Samuel, Phys. Rev. D 40, 1886 (1989).

[16] V.A. Kostelecký and R. Potting, Nucl. Phys. B 359, 545 (1991).

[17] V.A. Kostelecký and S. Samuel, Phys. Rev. Lett. 66, 1811 (1991).

[18] V.A. Kostelecký and R. Potting, Phys. Lett. B 381, 89 (1996).

[19] V.A. Kostelecký and R. Potting, Phys. Rev. D 63, 046007 (2001).

[20] R. Bluhm, Lect. Notes Phys. 702, 191 (2006). 
[21] V.A. Kostelecký, ed., CPT and Lorentz Symmetry IV, World Scientific, Singapore, 2008.

[22] V.A. Kostelecký, ed., CPT and Lorentz Symmetry V (World Scientific, Singapore, 2011).

[23] S. Liberati and D. Mattingly, arXiv:1208.1071.

[24] O. Bertolami et al., Phys. Lett. B 395, 178 (1997).

[25] A. Connes, M. Douglas, and A. Schwartz, J. High Energy Phys. 02, 003 (1998).

[26] I. Mocioiu, M. Pospelov, and R. Roiban, Phys. Lett. B 489, 390 (2000).

[27] S.M. Carroll et al., Phys. Rev. Lett. 87, 141601 (2001).

[28] Z. Guralnik, R. Jackiw, S.Y. Pi, and A.P. Polychronakos, Phys. Lett. B 517, 450 (2001).

[29] C.E. Carlson, C.D. Carone, and R.F. Lebed, Phys. Lett. B 518, 201 (2001).

[30] A. Anisimov, T. Banks, M. Dine, and M. Graesser, Phys. Rev. D 65, 085032 (2002).

[31] R. Gambini and J. Pullin, Phys. Rev. D 59, 124021 (1999).

[32] C.P. Burgess, J. Cline, E. Filotas, J. Matias, and G.D. Moore, JHEP 0203, 043 (2002).

[33] J. Alfaro, H.A. Morales-Técotl, and L.F. Urrutia, Phys. Rev. D 66, 124006 (2002).

[34] G. Amelino-Camelia, Mod. Phys. Lett. A 17, 899 (2002).

[35] D. Sudarsky, L. Urrutia, and H. Vucetich, Phys. Rev. D 68, 024010 (2003).

[36] V.A. Kostelecký, R. Lehnert, and M. Perry, Phys. Rev. D 68, 123511 (2003).

[37] A.R. Frey, JHEP 0304, 012 (2003).

[38] F.W. Stecker, Astropart. Phys. 20, 85 (2003).

[39] J.D. Bjorken, Phys. Rev. D 67, 043508 (2003). 
[40] R. Myers and M. Pospelov, Phys. Rev. Lett. 90, 211601 (2003).

[41] J. Cline and L. Valcárcel, JHEP 0403, 032 (2004).

[42] N.E. Mavromatos, Nucl. Instrum. Meth. B 214, 1 (2004).

[43] C.D. Froggatt and H.B. Nielsen, Ann. Phys. (Leipzig) 14, 115 (2005).

[44] W.A. Christiansen, Y.J. Ng, and H. van Dam, Phys. Rev. Lett. 96, 051301 (2006).

[45] W.A. Christiansen, Y.J. Ng, D.J.E. Floyd, and E.S. Perlman, Phys. Rev. D 83, 084003 (2011).

[46] D. Mattingly, Phys. Rev. D 77, 125021 (2008).

[47] G. Amelino-Camelia, L. Freidel, J. Kowalski-Glikman, and L. Smolin, Phys. Rev. D 84, 084010 (2011).

[48] H.P. Robertson, Rev. Mod. Phys. 21, 378 (1949).

[49] R. Mansouri and R.U. Sexl, Gen. Rel. Grav. 8, 497 (1977).

[50] C.N. Will, Theory and experimentation in Gravitational Physics (Cambridge University Press, Cambridge, England, 1993).

[51] V.A. Kostelecký and C.D. Lane, J. Math. Phys. 40, 6245 (1999).

[52] D. Bao, S.-S. Chern, and Z. Shen, An Introduction to Riemann-Finsler Geometry (Springer, New York, 2000).

[53] V.A. Kostelecký, Phys. Lett. B 701, 470 (2011).

[54] V.A. Kostelecký, N. Russell, and R. Tso, Phys. Lett. B 716, 470 (2012).

[55] Y. Nambu, Phys. Rev. Lett. 4, 380 (1960).

[56] J. Goldstone, Nuov. Cim. 19, 154 (1961).

[57] J. Goldstone, A. Salam, and S. Weinberg, Phys. Rev. 127, 965 (1962).

[58] F. Englert and R. Brout, Phys. Rev. Lett. 13, 321 (1964).

[59] P.W. Higgs, Phys. Rev. Lett. 13, 508 (1964). 
[60] G.S. Guralnik, C.R. Hagen, and T.W.B. Kibble, Phys. Rev. Lett. 13, 585 (1964).

[61] P.A.M. Dirac, Proc. R. Soc. Lon. A209, 291, (1951).

[62] Y. Nambu, Prog. Theor. Phys. Suppl. Extra 190 (1968).

[63] J.D. Bjorken, Ann. Phys. 24, 174 (1963).

[64] R. Bluhm and V.A. Kostelecký, Phys. Rev. D 71, 065008 (2005).

[65] R. Bluhm, S.-H. Fung, and V.A. Kostelecký, Phys. Rev. D 77, 065020 (2008).

[66] T. Jacobson and D. Mattingly, Phys. Rev. D 64, 024028 (2001).

[67] P. Kraus and E.T. Tomboulis, Phys. Rev. D 66, 045015 (2002).

[68] J.W. Moffat, Intl. J. Mod. Phys. D 12, 1279 (2003).

[69] O. Bertolami and J. Paramos, Phys. Rev. D 72, 044001 (2005).

[70] B. Altschul, and V.A. Kostelecký, Phys. Lett. B 628, 106 (2005).

[71] J.L. Chkareuli et al., Nucl. Phys. B 796, 211 (2008).

[72] R. Bluhm, N. Gagne, R. Potting, and A. Vrublevskis, Phys. Rev. D 77, 125007 (2008).

[73] S.M. Carroll, T.R. Dulaney, M.I. Gresham, and H. Tam, Phys. Rev. D 79, 065011 (2009).

[74] M.D. Seifert, Phys. Rev. D 79, 124012 (2009).

[75] M.D. Seifert, Phys. Rev. D 81, 065010 (2010).

[76] O.J. Franca, R. Montemajor, L.F. Urrutia, Phys. Rev. D 85, 085008 (2012).

[77] V.A. Kostelecký and R. Potting, Gen. Rel. Grav. 37, 1675 (2005)

[78] V.A. Kostelecký and R. Potting, Phys. Rev. D 79, 065018 (2009).

[79] B. Altschul, Q.G. Bailey, and V.A. Kostelecký, Phys. Rev. D 81, 065028 (2010).

[80] S.M. Carroll and E.A. Lim, Phys. Rev. D 70, 123525 (2004).

[81] S. Kanno and J. Soda, Phys. Rev. D 74, 063505 (2006). 
[82] E.A. Lim, Phys. Rev. D 71, 063504 (2005).

[83] P.G. Ferreira, B.M. Gripaios, and R. Saffari, Phys. Rev. D 75, 044014 (2007).

[84] B. Li, D.F. Mota, and J.D. Barrow, Phys. Rev. D 77, 024032 (2008).

[85] T.R. Dulaney, M.I. Gresham, and M.B. Wise, Phys. Rev. D 77, 083510 (2008).

[86] J. Beltran Jimenez and A.L. Maroto, Phys. Rev. D 80, 063513 (2009).

[87] V.A. Kostelecký and C.D. Lane, Phys. Rev. D 60, 116010 (1999);

[88] V.A. Kostelecký, and N. Russell, Rev. Mod. Phys. 83, 11 (2011); arXiv:0801.0287.

[89] R. Bluhm, V.A. Kostelecký, and N. Russell, Phys. Rev. Lett. 79, 1432 (1997).

[90] R. Bluhm, V.A. Kostelecký, and N. Russell, Phys. Rev. D 57, 3932 (1998).

[91] H. Dehmelt et al.. Phys. Rev. Lett. 83, 4694 (1999).

[92] R. Mittleman et al., Phys. Rev. Lett. 83, 2116 (1999).

[93] G. Gabrielse et al.. Phys. Rev. Lett. 82, 3198 (1999).

[94] R. Bluhm and V.A. Kostelecký, Phys. Rev. Lett. 84, 1381 (2000).

[95] L.-S. Hou, W.-T. Ni, and Y.-C.M. Li, Phys. Rev. Lett. 90, 201101 (2003).

[96] B. Heckel et al., Phys. Rev. Lett. 97, 021603 (2006).

[97] B. Heckel et al.l, Phys. Rev. D 78, 092006 (2008).

[98] R. Bluhm, V.A. Kostelecký, and N. Russell, Phys. Rev. Lett. 82, 2254 (1999).

[99] D. Bear et al., Phys. Rev. Lett. 85, 5038 (2000).

[100] D.F. Phillips et al., Phys. Rev. D 63, 111101 (2001).

[101] M.A. Humphrey et al., Phys. Rev. A 68, 063807 (2003).

[102] F. Canè et al., Phys. Rev. Lett. 93, 230801 (2004).

[103] B. Altschul, Phys. Rev. D 75, 041301 (2007). 
[104] J.M. Brown et al., Phys. Rev. Lett. 105, 151604 (2010).

[105] C. Gemmel et al., Phys. Rev. D 82, 111901 (2010).

[106] B. Altschul, Phys. Rev. D 79, 061702 (2009).

[107] M. Smiciklas et al., Phys. Rev. Lett. 107, 171604 (2011).

[108] R. Bluhm et al., Phys. Rev. Lett. 88, 090801 (2002).

[109] R. Bluhm et al., Phys. Rev. D 68, 125008 (2003).

[110] B. Altschul, Phys. Rev. D 81, 041701 (2010).

[111] M.C. Fujiwara et al., ALPHA collaboration, in V.A. Kostelecký, ed., CPT and Lorentz Symmetry V (World Scientific, Singapore, 2011).

[112] V.A. Kostelecký and M. Mewes, Phys. Rev. Lett. 87, 251304 (2001).

[113] V.A. Kostelecký and M. Mewes, Phys. Rev. D 66, 056005 (2002).

[114] V.A. Kostelecký and M. Mewes, Phys. Rev. Lett. 97, 140401 (2006).

[115] V.A. Kostelecký and M. Mewes, Phys. Rev. Lett. 99, 011601 (2007).

[116] H. Müller et al., Phys. Rev. Lett. 99, 050401 (2007).

[117] F.R. Klinkhamer and M. Risse, Phys. Rev. D 77, 117901 (2008).

[118] S. Hermann et al., Phys. Rev. D 80, 105011 (2009).

[119] Ch. Eisele, A. Yu. Nevsky, and S. Schiller, Phys. Rev. Lett. 103, 090401 (2009).

[120] M.E. Tobar et al., Phys. Rev. D 80, 125024 (2009).

[121] M.A. Hohensee et al., Phys. Rev. D 82, 076001 (2010).

[122] B. Altschul, Phys. Rev. D 80, 091901 (2009).

[123] J.-P. Bocquet et al., Phys. Rev. Lett. 104, 241601 (2010).

[124] S. Parker et al., Phys. Rev. Lett. 106, 180401 (2011).

[125] S.M. Carroll, G.B. Field, and R. Jackiw, Phys. Rev. D 41, 1231 (1990). 
[126] S. Coleman and S.L. Glashow, Phys. Rev. D 59, 116008 (1999).

[127] S.T. Scully and F.W. Stecker, Astroparticle Phys. 31, 220 (2009).

[128] X.-J. Bi et al., Phys. Rev. D 79, 083015 (2009).

[129] K. Greisen, Phys. Rev. Lett. 16, 748 (1966).

[130] G.T. Zatsepin, V.A. Kuzmin, in Cosmic rays, Moscow, No. 11, p. 45 - 47, vol. 11 (1969), vol. 11, pp. 4547.

[131] V.A. Kostelecký, Phys. Rev. Lett. 80, 1818 (1998);

[132] V.A. Kostelecký, Phys. Rev. D 61, 016002 (2000);

[133] V.A. Kostelecký, Phys. Rev. D 64, 076001 (2001).

[134] J.M. Link et al., FOCUS Collaboration, Phys. Lett. B 556, 7 (2003).

[135] B. Aubert et al., BaBar Collaboration, Phys. Rev. Lett. 100, 131802 (2008).

[136] A. Di Domenico, KLOE collaboration, Found. Phys. 40, 852 (2010).

[137] V.A. Kostelecký and R.J. Van Kooten, Phys. Rev. D 82, 101702 (2010).

[138] R. Bluhm, V.A. Kostelecký and C.D. Lane, Phys. Rev. Lett. 84, 1098 (2000).

[139] V.W. Hughes et al., Phys. Rev. Lett. 87, 111804 (2001).

[140] G.W. Bennett et al., Phys. Rev. Lett. 100091602 (2008).

[141] D. Colladay and V.A. Kostelecký, Phys. Lett. B 511, 209 (2001).

[142] M.A. Hohensee et al., Phys. Rev. D 80, 036010 (2009).

[143] V.M. Abazov et al. (D0 Collaboration), Phys. Rev. Lett. 108, 261603 (2012).

[144] B. Charneski et al., Phys. Rev. D 86, 045003 (2012).

[145] V.A. Kostelecký and M. Mewes, Phys. Rev. D 69, 016005 (2004).

[146] V.A. Kostelecký and M. Mewes, Phys. Rev. D 70, 031902(R) (2004).

[147] V.A. Kostelecký and M. Mewes, Phys. Rev. D 70, 076002 (2004). 
[148] L.B. Auerbach et al., LSND Collaboration Phys. Rev. D 72076004 (2005).

[149] J.S. Diaz, V.A. Kostelecký and M. Mewes, Phys. Rev. D 80, 076007 (2009).

[150] R. Abbasi et al., IceCube Collaboration Phys. Rev. D 82112003 (2010).

[151] P. Adamson et al., MINOS Collaboration Phys. Rev. Lett. 105151601 (2010).

[152] J.S. Diaz, and V.A. Kostelecký, Phys. Lett. B 700, 25 (2011).

[153] J.S. Diaz, and V.A. Kostelecký, Phys. Rev. D 85, 016013 (2012).

[154] V. Barger, D. Marfatia, and K. Whisnant, Phys. Lett. B 653, 267 (2007).

[155] T. Katori, MiniBooNE Collaboration, in V.A. Kostelecký, ed., CPT and Lorentz Symmetry V (World Scientific, Singapore, 2011).

[156] Q.G. Bailey and V.A. Kostelecký, Phys. Rev. D 74, 045001 (2006).

[157] J.B.R. Battat, J.F. Chandler, and C.W. Stubbs, Phys. Rev. Lett. 99, 241103 (2007).

[158] K.-Y. Chung et al., Phys. Rev. D 80, 016002 (2009).

[159] H. Müller et al., Phys. Rev. Lett. 100, 031101 (2008).

[160] V.A. Kostelecký and J. Tasson, Phys. Rev. Lett. 102, 010402 (2009).

[161] V.A. Kostelecký and J. Tasson, Phys. Rev. D 83, 016013 (2011).

[162] M.A. Hohensee et al., Phys. Rev. Lett. 106, 151102 (2011).

[163] L. Iorio, Class. Quant. Gravit. 29, 175007 (2012).

[164] V.A. Kostelecký, N. Russell, and J. Tasson, Phys. Rev. Lett. 100, 111102 (2008).

[165] V.A. Kostelecký and M. Mewes, Phys. Rev. D 80, 015020 (2009).

[166] V.A. Kostelecký and M. Mewes, Phys. Rev. D 85, 096005 (2012). 